\title{
Human Navigation in a Multilevel Travelling Salesperson Problem
}

\author{
Panagiotis Mavros ${ }^{* 1}$, Michael van Eggermond ${ }^{1,3}$, and Christoph Hölscher ${ }^{1,2}$ \\ ${ }^{1}$ Future Cities Laboratory, Singapore ETH Centre, Singapore \\ ${ }^{2}$ Chair of Cognitive Science, ETH Zürich, Switzerland \\ ${ }^{3}$ Mobility \& Transportation, Institute of Civil Engineering, University of Applied Sciences \\ (FHNW), Muttenz, Switzerland
}

January 18,2022

\begin{abstract}
Finding the optimal tour that visits a series of locations sequentially, such as going for errands, is an everyday task formally known as the travelling salesperson problem (TSP). In this article we focus on the understudied type of multilevel or M-TSP, which take place in a multilevel environment, like a building. In a TSP, the number of alternative tours the decision-maker needs to consider is given by the factorial of the locations to visit; hence a 3-target TSP has 6 alternatives and a 12target TSP has 479 million. Considerable research has focused on combinatorial optimisation algorithms for TSPs, and in the cognitive sciences there has equally been a sustained interest on how various foraging species and humans achieve remarkably optimal performance. However, research has primarily studied planar environments, and it is unclear how people will combine horizontal and vertical spatial information to make navigational decisions in a multilevel TSP.

In this study, we asked 41 participants to first learn the locations of 12 shops (targets) in a multilevel building, and then complete a structure mapping task and two open 8-target M-TSP tasks (more than 40.000 alternatives). Using bayesian methods for mixed effects modelling, we show that human performance in navigational M-TSPs is lower than this of Euclidean TSPs, and we differentiate between the choice of tour (visit sequence) and transitions (local wayfinding). Our results show an effect of horizontal versus vertical learning. We also found that performance in navigational TSP are a composite of global and local decision making, and the people adaptively employ a path-based, rather than euclidean, measure of distance when this is ecologically relevant. Overall we provide multiple sources of evidence for the horizontal bias theory both in mental representations and wayfinding behaviour.

This study contributes to current knowledge of mental representations 3D space and is the first to provide human data on an multilevel TSP. More generally, these findings have implications for our understanding of wayfinding and navigational behaviour in multilevel environments.

Keywords: navigation, travelling salesman, TSP, multilevel building, trip-chaining, spatial decision making, strategies
\end{abstract}

*Corresponding author, email: panos.mavros@sec.ethz.ch 


\section{Introduction}

Imagine running some errands in the city-centre or visiting a few shops located on different floors of a shopping mall. Both are typical and everyday examples of the travelling salesperson problem (TSP); for example up to $60 \%$ of pedestrian shoppers visit 3-8 locations (Borgers \& Timmermans, 2014) . The TSP is concerned with finding the shortest tour that visits a number of locations (traditionally called 'cities' in reference to the origins of the problem) that are distributed in space. These locations can be distributed along a topographical plane, a network, or as in the present study, in vertical, multi-level environments. Although human behaviour to solve TSP has been studied for decades, TSP that involve navigation across multiple floors such as large buildings, which we define as the multilevel-TSP or M-TSP, has been neglected.

For a number of locations $a, b, c, d, e$ the 'shopping list' can be visited in many different orders (e.g. $a b c d e, b a e d c$, etc.) which are not equal in terms of overall distance travelled. Formally, the number of possible solutions for a TSP of $n$ number of locations to visit, can be computed as the factorial of $n$ or $\frac{[n-1] !}{2}$. Therefore for a 3-city TSP the decision-maker has to evaluate 6 solutions, for an 8-city TSP 40320, for a 12-city TSP has 479 million solutions, and a 50-city has $3.04 \times 10^{64}$ alternative solutions. Despite the computational demands associated with solving a TSP, both humans and other species can routinely solve 20 - or 40-city TSPs which can consist of billions of alternative solutions (Macgregor \& Chu, 2011), and demonstrate remarkable performance by recurring only few minutes to find tours that are highly optimal, e.g. less than $10 \%$ longer than the optimal on average - often outperforming computational models in both speed and efficiency. For this reason, beyond their practical applications in transportation, operations research and computer science (Cook, 2011), TSPs are also of great interest for psychological research. TSP as part of understanding human and animal cognition and spatial behaviour, spatial decision making, and (spatial) heuristics. Moreover, as noted by Hales and colleagues, the TSP is a demanding cognitive task that dissociates between spatial memory and orientation on one hand, and spatial decision making on the other; thus it can be a promising tool to investigate other phenomena such as the mechanisms of Alzheimer's disease and Dementia (Hales et al., 2021).

More broadly, in many cities across the world, the development of high-rise buildings is increasing - especially in the South-East Asia where this phenomenon is labelled vertical cities (Lau \& Zhang, 2015). In cities like Hong Kong, Singapore and others this vertical 'sprawl' is coupled with the development of extensive interconnected pedestrian networks that connect multiple buildings and floors usually in central business district and transit oriented developments (Blauvelt et al., 2016; Zhang \& Chiaradia, 2020). There is increasing literature that individuals' mental representations of 3D spaces are processed differently along the horizontal axes than along the vertical axis, or vertical anisotropy (Brandt et al., 2015; Jeffery et al., 2013; Zwergal et al., 2016), and also that spatial navigation in multilevel environments presents unique challenges (e.g. Hölscher et al., 2006, 2009; Kuliga et al., 2019; Montello \& Pick, 1993; Passini, 1984). 3D mental representations are also involved in storing and expressing other types of information, such as time (Starr \& Srinivasan, 2021). Therefore, a better understanding the cognitive process of navigation in multilevel urban networks for pedestrian movement (Chang, 2002; Hölscher et al., 2006; Li et al., 2019) can have implications for the design of buildings and the simulation of human movement in such buildings and pedestrian networks.

The present behavioural study is, to the best of our knowledge, the first study to examine human performance in a multilevel navigational TSP, and overall contributes to the existing scholarship of 
human spatial cognition and behaviour of 3D space and multilevel buildings.

\section{Background}

\subsection{The travelling salesperson problem}

In computer science and operations research, the TSP belongs to the family of combinatorial optimisation problems (Christofides, 1975). Because the computational complexity of TSP increases exponentially with the size of the problem, exhaustive search (computing all alternatives) is the only way to find the optimal tour; it is considered an 'NP-complete' problem which cannot be solved in polynomial time (Macgregor \& Chu, 2011). TSP algorithms are well-studied due to numerous practical applications, in transport planning, from routing of school buses to delivering packages, but also in manufacturing, circuit board printing, warehousing and others(Cook, 2011). Most organisms routinely solve TSPs as part of their everyday behaviour - humans go for various errands, while bees, rats and chimpanzees forage for food - and all species aim to both minimise energy expenditure that is associated with distance travelled and maximise harvest (Cramer \& Gallistel, 1997). In non-human research, TSP are encountered as part of studies of optimal foraging on primates like chimpanzees (Menzel, 1973), bearded sakis (Shaffer, 2014), rats (Jovalekic et al., 2011), insects like pollinating bumblebees (Reynolds et al., 2013), and other species. For instance, chimpanzees use systematic optimal foraging strategies to minimise distance walked and maximise their yield during food gathering from up to 18 sources (an 18-point TSP has $6.4^{*} 10^{15}$ solutions), and can forage on widely distributed discrete resources.

In human research, TSP have been in the studied in the context of spatial cognition and navigation (Gärling \& Gärling, 1988; Hayes-Roth \& Hayes-Roth, 1979; Hirtle \& Garling, 1992; Kurose et al., 2001; Saisa \& Garling, 1987; Wiener et al., 2009). TSPs can be distinguished into open and closed, depending on either returning to the starting point in the case of a closed-TSP, or without returning to the starting point, in the case of open-TSP (or hamiltonian-path). Closed and open TSPs consist of a similar level of complexity (i.e. number of cities to visit), but individuals perform worse in openTSP (Chronicle et al., 2006), although this also depends on the spatial configuration of the points to be visited. Another important distinction is between visual and navigational versions of the TSP (Blaser \& Wilber, 2013; Wiener \& Tenbrink, 2008). Best described using Montello's taxonomy of spatial scales 1993, visual TSPs, take place in figural space, where all points can be perceived from a single location, are typically $2 \mathrm{D}$ and consist of abstract stimuli (e.g. dots on a computer screen), and the points can be connected with a direct line (Chronicle et al., 2006; Macgregor \& Ormerod, 1996). In cases when all targets (cities) are points on a euclidean plane, the problem is referred to as euclidean-TSP, or E-TSP (Haxhimusa et al., 2011; MacGregor, 2017). On the other hand, navigational TSPs require the person to physically walk in vista space (e.g. a room) or large-scale space (e.g. a building or even city); thus the targets may not always be visible and decision-makers need to rely on their spatial working memory(Wiener \& Tenbrink, 2008). Therefore, the scale of space as well as the setting have specific spatial memory requirements. Garling (1989) showed that compared to a spatial memory-only condition, access to a spatial representation like a map facilitates minimisation of the TSP tour, as participants can more easily take into account the spatial relationships between locations. Despite these differences, people have similar performance in figural and navigational TSPs (albeit in room-scale), employing similar, although not identical, strategies to 
solve the TSP (Blaser \& Wilber, 2013). In navigational TSPs taking place in room-scale environments (Blaser \& Wilber, 2013; Wiener et al., 2009) participants can walk directly from one point to another; whereas navigational TSPs in large scale spaces like urban environments (Gärling \& Gärling, 1988; Saisa \& Garling, 1987) or buildings involve additional spatial decision making required to choose between alternative paths, corridors or streets, connecting the various locations. Accordingly, Hui et al. (2009) argue, in navigational TSP the overall performance (distance walked) is comprised of two components of error: (a) the choice of tour or the order of visit (e.g. for 5 targets abcde the optimal tour is adceb, so acbde leads to excess distance) and (b) the excess distance of each transition, i.e. during wayfinding between each pair of locations in the tour (e.g. $a d, d c, c e, e b$ ) .

Both humans and other species make use of different strategies and heuristics to find solutions to TSPs. Some of the heuristics that have been proposed include the nearest-neighbour heuristic (or local minimising distance in Saisa \& Garling, 1987)), the $n$-step look ahead (Anderson, 1983), convex hull and convex hull with cheapest insertion criterion (Macgregor \& Ormerod, 1996), crossing avoidance, region-based planning (Wiener \& Mallot, 2003b), hierarchical clustering of proximal targets (Kirsch, 2012; MacGregor, 2015). Some of these heuristics, such as the convex hull and the hierarchical clustering that are typically studied with visual TSP and euclidean TSPs, and are thought to rely on the perceptual processes underlying the visual system (Macgregor \& Ormerod, 1996). These have also encouraged analogous algorithmic approaches based on hierarchical clustering (e.g. Haxhimusa et al., 2011; Kirsch, 2012). Depending on the configuration of targets in space, some heuristics may produce better results than others, and individuals generally switch adaptively to heuristics congruent with the task (e.g. if the targets form clusters or convex hull Blaser \& Wilber, 2013; Saisa \& Garling, 1987; Wiener et al., 2009), although performance drops with increased complexity in the arrangement. Nevertheless, there is a lack of studies on how people employ such heuristics and strategies when they navigate in large-scale environments, especially in buildings.

\section{$2.22 \mathrm{D}, 3 \mathrm{D}$ and M-TSP}

The majority of TSP studies with humans involve planar TSPs, for example connecting points on a screen or sheet of paper (Gärling \& Gärling, 1988; Macgregor \& Ormerod, 1996), collecting items inside a room Blaser \& Wilber (2013); Wiener et al. (2009) or even running for errands in a city (Saisa \& Garling, 1987). For this reason, the TSP was originally formulated in the context of visiting multiple cities or multiple locations within a city. However, there are many cases in everyday life where TSPS must be solved in 3D space. For instance this can occur while visiting complex multilevel buildings, from shopping malls to hospitals, or, for non-human species, foraging for food in the branches of a tree or a coral reef. These are general cases of 3D-TSPs, and it appears that people are generally able to solve TSPs in three-dimensional space, visiting locations by moving both in the horizontal and the vertical domains. As noted by Haxhimusa et al. (2011), an implication of 3D TSPs is that several TSP heuristics are not applicable. For example, there is no obvious extension of the convex-hull heuristic to $3 \mathrm{D}$ space, as the convex-hull in 3D forms a surface rather than a tour, and similarly, the crossing avoidance heuristic is also less applicable as self-intersecting lines are less likely to occur if points are distributed in three dimensions (Haxhimusa et al., 2011). Few studies have explored this topic in terms of human or non-human behaviour. In a series of experiments on rat behaviour in three-dimensional space, (Jovalekic et al., 2011) used a wooden lattice with 25 food locations to study rat foraging in multilevel environment. They showed that rats exhibit a horizontal bias (preference for horizontal movement) and solving first the vertical component of a downward path to a fixed reward 
location (Jovalekic et al., 2011); this is equivalent to the floor strategy observed in humans (Hölscher et al., 2006), although the rat behaviour is not discussed further in terms of TSP. Haxhimusa and colleagues conducted an experiment of 3D TSP behaviour, asking participants to solve a 3D TSP in a virtual space. The experiment consisting of spheres with diameter of 15 centimetres were generated within a cube $3 \times 2 \times 3$ meters, and TSP problems were generated by randomly selecting 6, 10, 20, and 50 spheres. They noted that TSP performance was lower than on 2D TSP of equal complexity (i.e. same number of cities) and also that participants considered the task more difficult, unnatural and had lower confidence on the quality of their solutions; however, their study is limited by the small number of participants. Phillips \& Layton (2010) conducted a TSP with a vertical component, but this was produced by a undulating terrain.

Movement in buildings is distinct from these cases, as vertical movement is discrete and constrained on different floors (or levels), hence here we focus on multilevel travelling salesman problems, or M-TSPs. To the best of our knowledge, M-TSP in multilevel environments has not been examined before in humans. In such a scenario, euclidean and path distance may be fully dissociated, for example a location on a floor above or below may be physically close but require a long detour to reach the stairs. Furthermore, M-TSPs offer the opportunity to study the presence of a horizontal or vertical bias, in other words if people will prefer visiting all locations on a floor before moving to other floors, or aim to complete all vertical movements first. In particular, it has been shown that people organise spatial information and navigate taking into account that locations can be distributed in regions (Manley et al., 2015; Wiener \& Mallot, 2003b), which is often referred as 'regionalisation'. Additionally, Büchner et al. (2007) demonstrated that people regionalize space in horizontal (i.e. by floor) and vertical domain. The M-TSP paradigm provides an experimental framework to study bias for targets on the horizontal and vertical regions. Finally, one theory proposed to explain the horizontal bias across species is the energetic requirements of vertical movement against the axis of gravity Nardi et al. (2012). To disentangle effort from vertical movement, researchers have user rotated lattice environments to study rat behaviour Grobéty \& Schenk (1992); Jedidi-Ayoub et al. (2021). Comparing behaviour in buildings allows to design experiments that control for the effect of physical effort required to move vertically, either by staircases (effortful vertical movement) or by escalators (effortless vertical movement). This offers a rare opportunity to disentangle whether the horizontal bias is physical (i.e. effort-related), situational (i.e. depends on the behavioural environment), or psychological (i.e. constant trait).

Mental representations of 3D space Solving navigational TSPs in large-scale buildings requires access to long term memory (cf. Wiener \& Tenbrink, 2008), thus here we turn to the issue of mental representations of three-dimensional spaces. In recent years there is an increasing number of studies addressing how organisms (insects, animals or humans) perceive and mentally represent vertical spaces and navigate in 3D space (e.g. Büchner et al., 2007; Davis et al., 2018; Grieves et al., 2021; Jeffery et al., 2013; Krukar et al., 2021; Lu \& Ye, 2019; Nardi et al., 2012). The perception and mental representation of 3D space is of important theoretical interest for spatial cognition due to the physical and qualitative differences of moving along the axis of gravity, in incline or vertically, "which poses constraints on affordances and energetic potential" (Nardi et al., 2012).

Recent studies consistently find that mental representations of 3D spaces exhibit anisotropic properties, specifically that vertical distances are underestimated as compared to horizontal distances and that many species exhibit a horizontal bias (preference for moving on the horizontal plane). This is 


\section{Schematised differences in TSPs.}

O Targets $\$ start $\square$ Obstacles Corridors

A Euclidean (visual) TSP

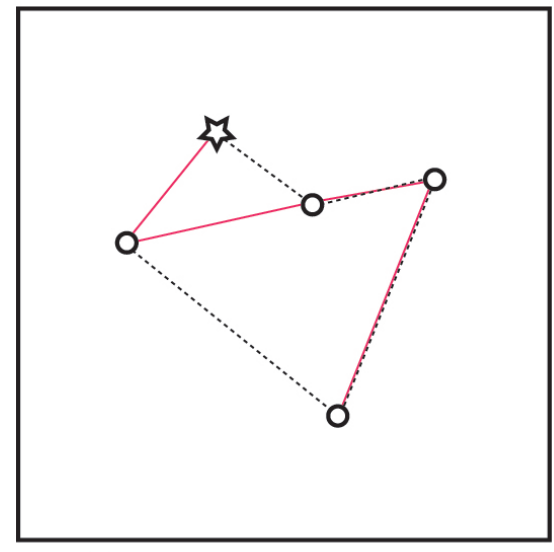

- Shortest tour

....... NN tour
B navigational TSP

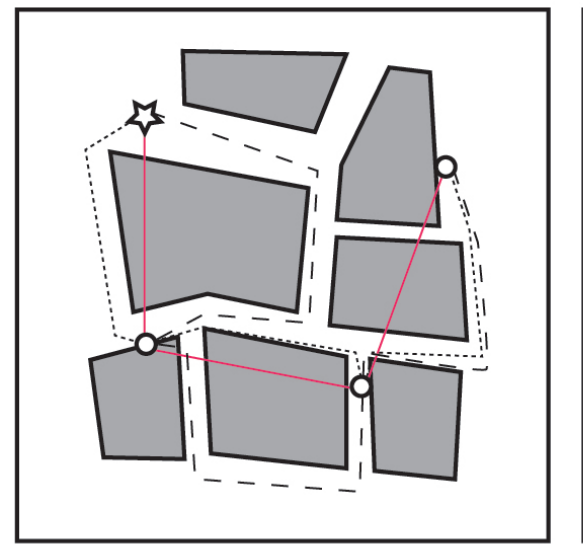

- Shortest tour

....... Shortest tour / shortest paths

- - Shortest tour / walked paths
C multilevel TSP

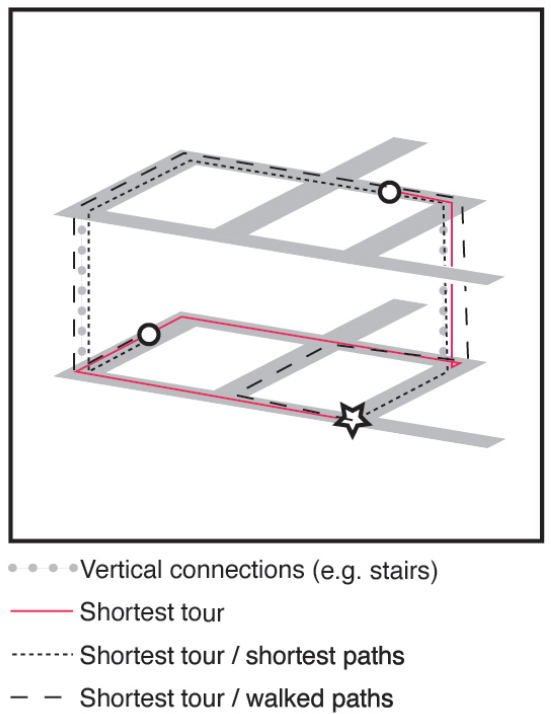

Figure 1: Diagram of key differences between visual, navigational and multilevel TSPs. (A) In a visual TSP (also known as euclidean TSP) all targets are on the same 2D plane (e.g paper, screen, room) and are always visible (Wiener \& Tenbrink, 2008). The strategy of always choosing the nearest neighbour (NN) does not always result in the shortest tour (Garling, 1989). (B) In navigational TSPs, in virtual reality or real-world, the targets are not always visible (Wiener \& Tenbrink, 2008), and obstacles in the environment (e.g. furniture in a room, corridor layout, buildings) require the person to also navigate during the tour. Therefore, the person may complete a TSP in the optimal tour (target sequence) but take sub-optimal (longer) paths between targets, called transitions. (C) In a multilevel TSP, targets are distributed in two or more planes of movement -typically building floors. In this example, the nearest neighbour is on a different level and the NN strategy produces the shortest/optimal tour, but a bias to stay on the same floor (region) or delay vertical movement, will result in a longer tour. Building upon research on how people regionalise space (Wiener \& Mallot, 2003b) in horizontal (i.e. by floor) or vertical domain (Büchner et al., 2007), M-TSPs provide an experimental framework to study bias for targets on the horizontal and vertical regions.

observed in animals Jedidi-Ayoub et al. (2021); Jovalekic et al. (2011) as well as people. Montello \& Pick (1993) observed that individuals perform better in distance estimation and pointing towards locations on the same floor compared to locations on the other floor. However, Lu \& Ye (2019) found that participants can achieve high levels of accuracy on a 3D pointing task, as long the environment afford direct visual access between floors (e.g. through atria). In other studies, participants overestimated vertical distances, either by walking up or down a staircase (Hanyu \& Itsukushima, 2000) or by 3D pointing (Brandt et al., 2015; Wilson et al., 2004). These differences appear even at the neural level, for instance recent results suggest that in rats, the grid-cells firing patterns reveal that vertical grid fields are ellipsoid, rather than hexagonal, and also are sparser, larger, irregular both in size and arrangement (Grieves et al., 2021).

A common aspect of most of these studies was that participants learned the buildings moving in each floor. Thus, the predominance of horizontal movement, and lack of direct visual access could be the underlying reason behind the difficulty to establish vertical relationships (Dollé et al., 
2015). However, in studies where the exposure to the environment during the learning phases is systematically controlled to be either vertical or horizontal, it is shown that individuals can flexibly use salient structures such as floors and staircases to organise their mental representations (e.g Büchner et al., 2007). For example, Dollé et al. (2015) asked participants to explore (guided learning) a virtual cylindrical three-level environment through either horizontal or vertical movement (floorlearners or column-learners). During testing they had to infer which object was in a room adjacent, below or above their location. Floor-learners failed to infer the location of objects on other floors, but, in contrast, column-learners were able to infer adjacent objects (Dollé et al., 2015). This suggests that integration of vista spaces is facilitated at the horizontal plane, and vertical movement does not impede the process of identifying spatial relationships (adjacency or shortcut), but integration of spatial knowledge is difficult across floors, in line with earlier findings by Montello \& Pick (1993).

The majority of studies have relied on 3D pointing and 3D cognitive distance estimation to investigate mental representations of space per se. However, an important function of mental representations of space is to guide navigation behaviour (Downs \& Stea, 1973; Golledge, 1999). Wayfinding studies in multilevel buildings have illustrated the additional cognitive challenges of vertical movement and movement across floor levels Feng et al. (2022); Gath-Morad et al. (2021); Hölscher et al. (2006); Kuliga et al. (2019), which arise both the spatial and cognitive constraints.

In this context, in order to solve an M-TSP task between targets that span multiple levels, navigators must judge distance and other spatial relationships (clustering, overlap) across both the horizontal and vertical domains. Thus, M-TSPs present the opportunity to study spatial decision making to disentangle how people evaluate and weight vertical versus horizontal distance in naturalistic task.

\subsection{Route and destination choice}

In parallel, there is a large scholarship from transport planning research looking at pedestrian route choices (although they have received less attention that of drivers' or public transport), and destination choice. An early study by Seneviratne and Morrall (Seneviratne \& Morrall, 1985) found that primarily pedestrians aim to minimise distance walked, while more recent work has shown the importance of other factors such as safety from traffic (Agrawal et al., 2008), pedestrian-friendly environments Guo (2009). Studies also show that pedestrians consider trade-offs between distance and other parameters, for example walking slightly more distance (detour) to pass from more attractive facilities (Salazar Miranda et al., 2021). Few studies have also addressed sequential decision making (Borgers \& Timmermans, 2014; Saisa \& Garling, 1987), showing that pedestrians are sensitive to distance and apply heuristics adaptively to reduce the total distance walked, and often use the optimal sequence but deviate from the optimal path. To conclude, most studies address route choice in outdoor settings, while there is a lack of route choice in indoor settings and in multilevel environments, especially in sequential decision making (TSP).

\subsection{The present study}

In the present article we present a novel, naturalistic M-TSP experiment that was completed in a real-world building. The study has three objectives. First, to study the structure of mental representations of $3 \mathrm{D}$ space and test the potential influence of learning process on spatial memory. In previous research participants learned and navigated a linear building (i.e. in an abstracted manner the building can be walked in $X$ and $Z$ but not $Y$, for example see Büchner et al., 2007). In contrast, 
in the present study the building corridors extends in $\mathrm{X}, \mathrm{Y}, \mathrm{Z}$ axes and forms a topological loop, which may potentially be more difficult to both perceive and externalise mental representations of multilevel spaces (Figure2). Second, we employ a naturalistic multilevel TSP task to understand navigation in complex multilevel environments. Path-planning to multiple targets requires knowledge about the length of different alternatives. Prior studies suggest an anisotropy of distance, such that vertical distance is overestimated. Third, by manipulating the mode of spatial learning into either horizontal or vertical (see Section 3), we investigate whether the way of learning locations in the horizontal or or vertical domain introduces a biases on the completeness and form of mental representations as well as wayfinding decisions.

To test these questions, we have developed a novel experiment that involves a multilevel travelling salesperson problem in a real-world large-scale building, paired with a spatial learning that biases horizontal or vertical movement. In order to assess the effects of learning we manipulated how participants learned the testing environments and target locations (Büchner et al., 2009; Dollé et al., 2015; Thibault et al., 2013). Participants learned the environment either by floor or by escalator (Figure 4). To summarise, the three objectives above can be formulated in the following hypotheses:

1. Horizontal training will provide a better understanding of the spatial configuration of each floor (more accurate mental representations).

2. Vertical training will provide a better understanding of the spatial configuration in the vertical domain (more accurate mental representations in terms of vertical relationships).

3. Horizontal training will reinforce horizontal bias.

4. Vertical training strategy will reduce the horizontal bias.

\section{Materials and Methods}

\subsection{Participants}

A total of 41 participants (21 female; mean age 24.5 years old, range: 20 - 35) took part in this experiment, while one was excluded from analysis due to technical issues during data collection. Participants were recruited from the participant pools of local universities of Singapore, with criteria for inclusion: to be able and willing to walk continuously for up to 1.5 hours. All participants were compensated 20.00 SGD (approx. 15 USD) for their participation. The experiment design and procedure was approved by the ETH Ethics Committee (EK-2016-N-70). All methods were performed in accordance with the Declaration of Helsinki. Table 1 shows descriptive statistics for the participant sample.

The experiment adopted a simple factorial design, varying the factor training with two levels: horizontal and vertical. First, participants learned the layout and the location of twelve (12) target destinations (shops) in large, complex, multilevel building (shopping mall) through a guided-tour performed by a researcher. Figure 4 shows the two variations of the training tour. The aim was to manipulate how participants integrated horizontal and vertical spatial knowledge (Büchner et al., 2007; Dollé et al., 2015) and explore whether increased vertical movement can influence individuals' horizontal bias. For the horizontal training, participants were guided to all the target locations on each floor completing a full loop of the floor - before going to the next floor, and used the same block of escalators for all vertical transitions. After visiting all the targets, they walked the entire route backwards, 
Table 1: Participant Characteristics

\begin{tabular}{llll}
\hline Characteristic & Overall, N =41 & Horizontal, N = 22 & Vertical, N=19 \\
\hline Age & $24.5(3.2)$ & $24.3(2.8)$ & $24.7(3.6)$ \\
Sex & & & \\
Female & $21 / 41(51 \%)$ & $11 / 22(50 \%)$ & $10 / 19(53 \%)$ \\
Male & $20 / 41(49 \%)$ & $11 / 22(50 \%)$ & $9 / 19(47 \%)$ \\
SBSOD & $3.96(0.75)$ & $3.85(0.68)$ & $4.09(0.82)$ \\
Training start & & & \\
Level 1 & $20 / 41(49 \%)$ & $10 / 22(45 \%)$ & $10 / 19(53 \%)$ \\
Level 4 & $21 / 41(51 \%)$ & $12 / 22(55 \%)$ & $9 / 19(47 \%)$ \\
Familiar & $15 / 41(37 \%)$ & $8 / 22(36 \%)$ & $7 / 19(37 \%)$ \\
\hline Note: & Mean (SD); $\mathrm{n} / \mathrm{N}(\%)$ & &
\end{tabular}

visiting each target. This resulted in a total of six (6) level changes, 3 upwards and 3 downwards. In contrast, for the vertical training, participants were guided to the two nearest target locations left and right from each escalator and then switched floors; once visiting all the targets adjacent to an escalator, then they proceeded to the next escalator. This resulted in a total of twenty-two (22) level changes. In both conditions participants had the opportunity to approach, see and visit each target location from both sides of the corridor, similar to the systematic training method used in other studies (Howard et al., 2014).

\subsection{Experiment Design}

Testing took place immediately after the training phase. First, participants completed a structure mapping task, or SMT (Büchner et al., 2007). Second, they completed two M-TSP tasks to test their wayfinding behaviour and M-TSP solution strategies.

\subsubsection{Structure mapping task (SMT)}

Immediately after the training phase (guided tour) participants were asked to complete a structure mapping task (Büchner et al., 2007). This task aimed to assess the quality as well as the nature of participants' mental representation of the building and the twelve target locations. Previous studies have shown that this task allows to differentiate between mental representations of space with an emphasis on verticality such as overlapping relationships (e.g. columns of objects), versus those who structure their mental representations of space horizontally, e.g. in 'rows' or planes (Büchner et al., 2007).

\subsubsection{TSP navigation task}

For the TSP, the navigation task was devised as a 'shopping list' (Wiener et al., 2009). The list included eight (8) different destinations across four floors, including three destinations on floors 2 and 3 , and one destination on floors 1 and 4 (see also Appendix A). The destinations were selected so as to be naturally organised in vertical clusters (Figure 2). Participants completed two (2) consecutive M-TSP navigation tasks. Both tasks included the exact same set of target locations (shopping list), but starting from a different location on floor $2^{\text {nd }}$ or $3^{\text {rd }}$. As a result, the optimal solution was different 
for each task, yet we could observe if participants perform better in the M-TSP the second time they visit the same list.

Thus, the task allowed to test if decision making was influenced by regionalisation by floor (horizontally) or escalator / cluster (vertically) (Büchner et al., 2007).

\subsection{The testing environment}

The study was conducted in a major shopping mall in the Jurong East area of Singapore. The building consists of a seven-floor commercial podium (five (5) above-ground levels and two (2) below-ground levels), as well as an office tower of 20 storeys. In the present experiment, participants were restricted to only visit the four main floors of the mall (Levels $1,2,3 \& 4$; note that Level 1 corresponds to ground-floor). The shopping mall has a footprint of approximately $140 \times 108$ meters, covers an area of 15,120 sqm, Gross Floor Area (GFA) 55,177 sqm / 593,906 sqft, Net Lettable Area 38,208 sqm, and has more than 40 million visitors annually ${ }^{1}$. The building layout includes bi-directional escalators for vertical movement, situated in four different locations (Figure 4). For reproducibility, we should note that at the time of the experiment, one of the escalators did not reach the ground-floor (i.e. connected only levels L2,L3 and L4), but an additional escalator between L1 and L2 has been added recently.

\subsubsection{Target locations}

Figure 2 shows the distribution of the 12 targets inside the building. Level 1 and level 4 had 2 targets each, and level 2 and level 3 had 4 targets each. The destinations were selected so as to form horizontal and vertical clusters (see Appendix A for additional information). A horizontal cluster consisted of all locations in the same floor, while a vertical cluster is defined as all the locations that are on the same side of the building, around a salient feature of the building (e.g. around an atrium), clustered near the same vertical connector (near an escalator), or overlapping closely but across different floors (see Figure $2 \mathrm{C}$ ). Therefore, the selection of target locations create horizontal-vertical conflict, where a participant could prioritise to visit all locations in the same floor before proceeding to the next (horizontal bias), to visit all the locations in the same vertical cluster before going to the next cluster (vertical bias), or a mix of the two strategies. All 12 target locations were included in the structure mapping task, but a subset of 8 locations were included in the M-TSP Task: 1 in level 1, 3 in level 2, 3 in level 3, and 1 in level 4 (shown in bold circles in Figure $2 \mathrm{C}$ ).

\subsection{Procedure}

Figure 4 shows the order of experimental procedures and the main manipulation (training phase). The experimenter met participants individually, at a location in proximity but without direct view to the testing environment. They were told they would participate in a study to understand "study decision making while moving through a busy environment", answered potential questions, and obtained informed consent.

Training Phase Participants were given a guided-tour of the testing environment, to systematically obtain spatial knowledge of the building layout and the target locations (Figure 2). Participants

\footnotetext{
${ }^{1}$ Westgate: https://www.capitaland.com/sg/en/lease/mall-listing/westgate.html
} 


\section{Testing environment}

A Axonometric diagram

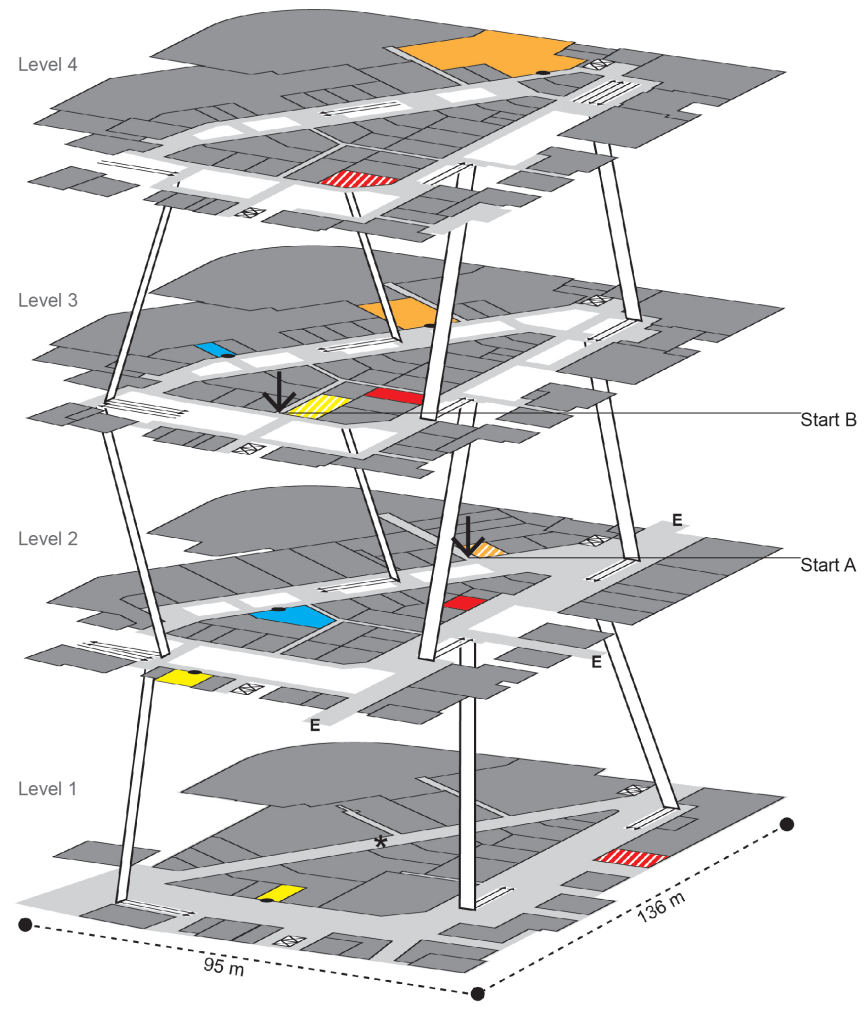

Walkable space

Built space

Escalators

\ Lifts (not used)

Targets, colour-coded by vertical cluster

VA Targets not used in the TSP task

E Elevated pedestrian network

* Escalator does not connect L1-L2
B Spatial network

Walkable spaces / corridors

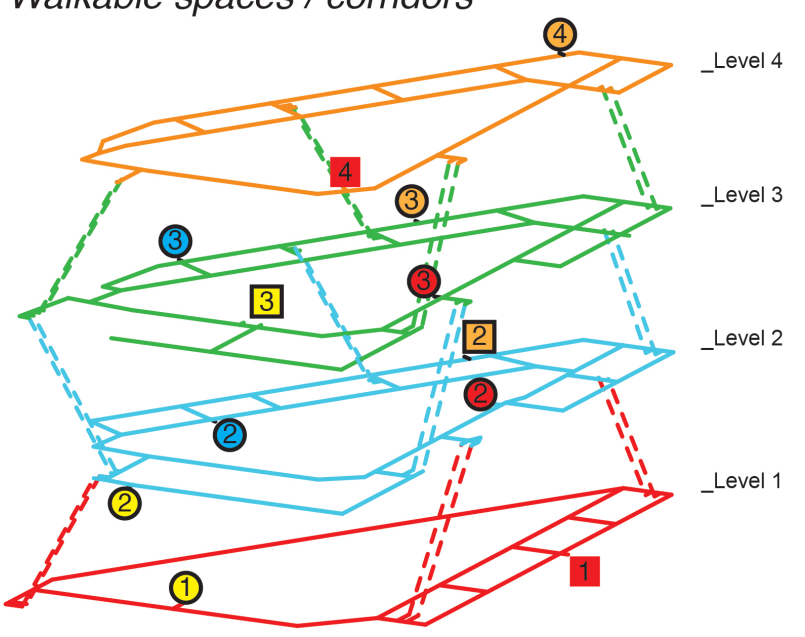

C Schematic top-view

Number indicates floor/level

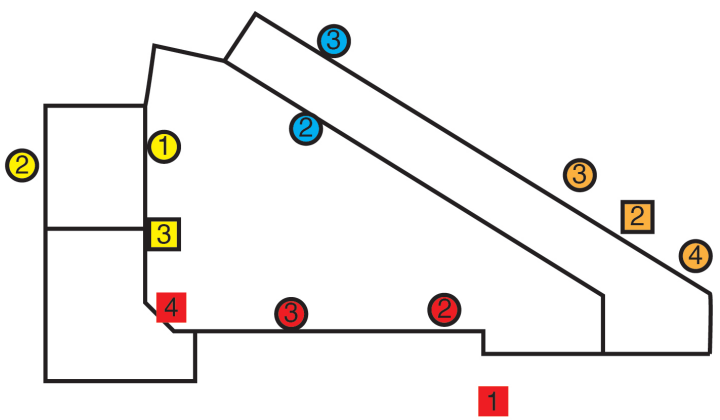

Targets, colour-coded by vertical cluster Targets / Start locations

Targets not used in the TSP task

Figure 2: The testing environment. (A) This axonometric view of the building shows the position of the 12 target locations, colour-coded according to the vertical cluster they were assigned. $(B)$ This schematised axonometric view of the building emphasises the spatial network, i.e. the centre-line of each corridor that participants were allowed to walk. Participant trajectories were digitised using this spatial network. (C) This floor-plan shows the top-view position of the 12 target locations, colourcoded for cluster. Marked with black outline are the eight (8) targets used for the TSP. 

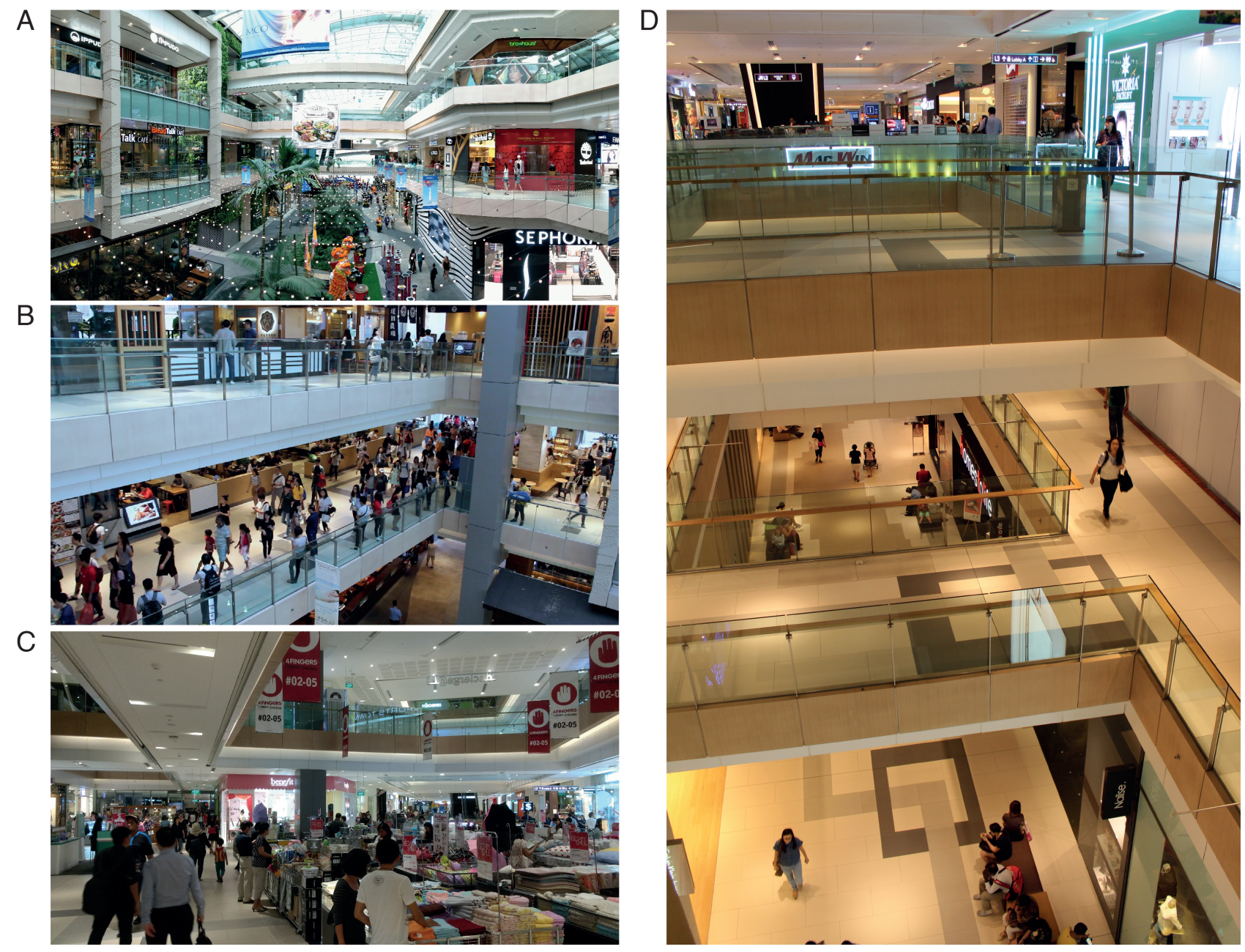

Figure 3: Images of the testing environment. Note that individual shops may have changed since. (A) View of the main atrium, taken from Level 2. (B) View of Levels 1, 2 and 3. (C) View of Levels 2 and 3, taken from the Level 2. (D) View of Levels 1,2 and 3, taken from Level 3. 


\section{A Procedure}

\section{Prior}

Recruit. \&

Screening

Testing session

$\rightarrow$\begin{tabular}{l|l|} 
Informed \\
Consent
\end{tabular}$\rightarrow \rightarrow \begin{aligned} & \text { Spatial } \\
& \text { learning }\end{aligned}$

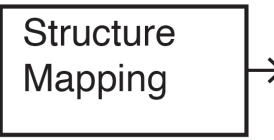

$x 2$

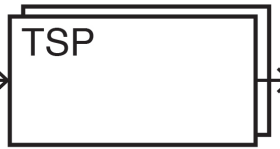

Questionnaires

\section{B Spatial learning}

Horizontal (floor by floor)

Vertical (escalator by excalator)
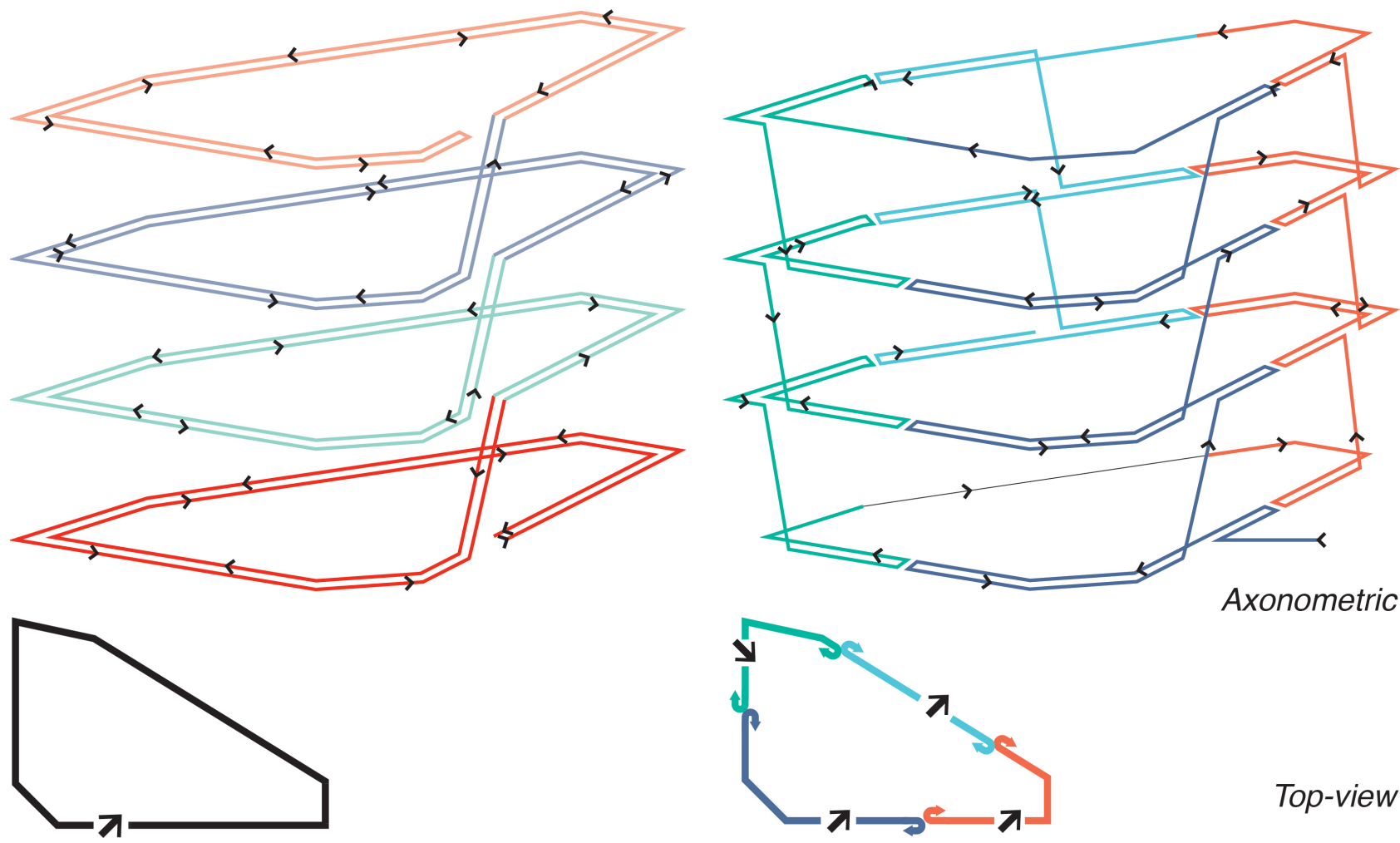

Axonometric

Figure 4: Experimental procedures. (A) Order of experimental procedures. The entire experiment took approximately $1-1.25$ hours. (B) The main manipulation consisted of two different types of training (spatial learning), either horizontal, visit all targets in each floor before going to the next, or vertical visit targets adjacent to each escalator in all floors, then proceed to the next escalators. Once visiting all targets in one directions, the researcher guided the participant backwards, retracing the training route back to the start. In this way participants visited each target twice, approaching it from both sides.

were randomly assigned into two groups: horizontal (22 participants; 11 female) or vertical (19 participants, 10 female), and received a guided tour of the 12 locations used for the experiment as described in the Experiment Design (Section 3.2, see also Figure 4). To control for the potential anchoring effect of the starting location, we counterbalanced the starting level (L1 or L4) and which escalator-block used between participants.

The training phase took approximately 30 to 40 minutes to complete. The experimenter started from one of the four escalators, walking on each level to reach the nearest target location. Upon arrival in front of each target location, participants were briefly stopped, shown a card $(6 \times 6 \mathrm{~cm})$ with a photograph of the exact same location, and told to remember the place, the card and its name. Then, they either proceeded to the next target location: In the horizontal training, participants were taken to all targets on each floor before proceeding to the next level (horizontal training, Figure 4), whereas 
in the vertical training they were taken to all targets adjacent to each escalator - i.e. branching out, then moving to the next level/floor before; when all the targets adjacent to an escalator where visited, they proceeded to the next on the same level (vertical training; Figure 4). Once they reached all targets and floors, they retraced the guided-route in the exact opposite direction, and targets were pointed out for a second time but without stopping.

SMT Phase At the end of the training phase participants were guided to a separate location to complete the structure mapping task. While seated, they were provided a flat horizontal white board, measuring $38 \times 53.5 \mathrm{~cm}$. The twelve location cards (the same used during the training phase) were shuffled and given to the participant with the instruction "how do you think they are arranged in space". Participants were given as much time as needed to place and arrange the cards on the board (Figure 5). An image of the board was taken to be analysed later.

Navigation Phase During testing, participants completed two M-TSP tasks. They were led to the start location for their first TSP task (shown in Figure $2 \mathrm{C}$ with a rectangle label). There, the experimenter handed the list with the names of the targets (but not their image) to the participant with the instruction to visit all the locations on an 8-target list, in whatever order she/he found more 'efficient'. The same list and list order was given to all participants. The task ended when then participants had visited all the target locations once. At the end of each task, participants will be asked to complete the NASA TLX Hart \& Staveland (1988), and then they were then guided to the next starting location, where the repeated the same procedure. During wayfinding, the experimenter followed them at a close distance, recording their trajectory on the building floor plans.

Questionnaires and debriefing Finally, immediately after the second task, participants were led to a quiet location and asked to fill in a questionnaire about their spatial abilities and. Lastly, they were debriefed about the purpose of the experiment, thanked and reimbursed for their time and effort.

\section{Measures and analysis}

\section{Self-reported Measures}

Spatial Ability We measured participants' navigation ability using the Santa Barbara Sense of Direction scale (SBSOD; Hegarty et al., 2002). The SBSOD scale includes statements such as "I am very good at giving directions" or "I am very good at reading maps" and has consistently been found to predict navigational performance (Weisberg \& Newcombe, 2015). We note that due to a technical error, responses for the last 5 items of the questionnaire were not recorded (items 11 to 15); thus, the resulting score was computed as the average of items 1 - 10 (reverse scored as appropriate).

Mental demand To assess the subjective difficulty of the TSP, we asked participants to complete the NASA Task Load index (Hart \& Staveland, 1988), immediately after each task. The unweighted version of each scale was analysed separately (Hart, 2006) and below we focus specifically on the Mental Workload sub-scale. 


\section{Structure Mapping Task}

\section{Classification Scheme}

a

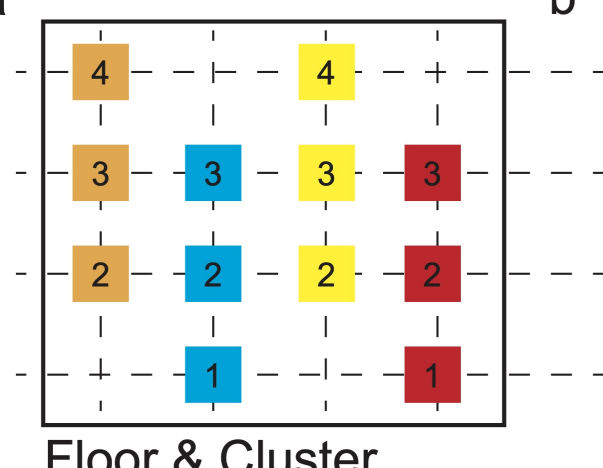

Floor \& Cluster b

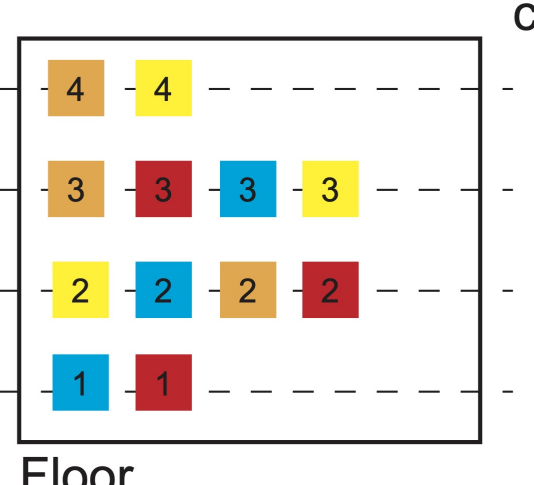

C

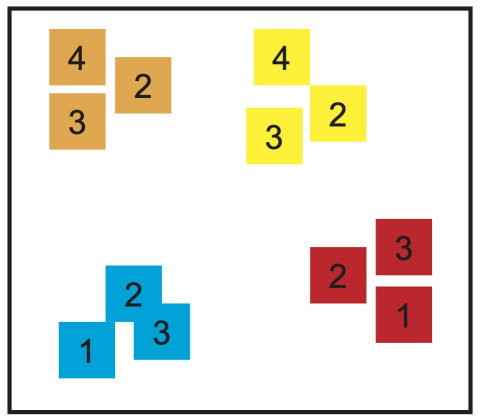

Survey
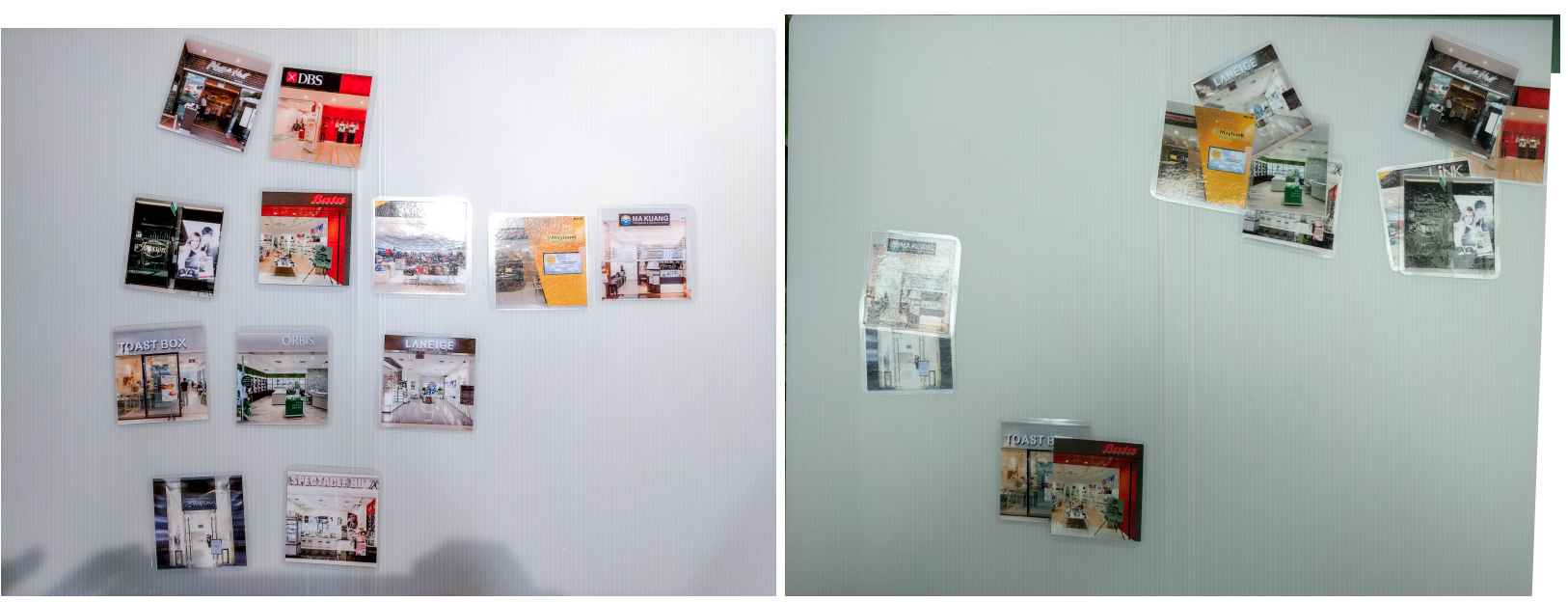

Figure 5: (Top) Diagram of the structure mapping task classification scheme. Each rectangle represents a unique location, colour represents a vertical cluster, and the number refers to the floor. In the floor $x$ cluster category (a), all locations are positioned on the correct floor and vertical relationships (i.e. vertical clusters) are aligned. In the floor category (b) locations that are on the same floor are organised as a row, but vertical relationships (i.e. between floors) are not aligned. In the survey category (c) the locations are arranged in a 'spatial' manner (top-view like) and vertical clusters are respected. (Bottom) Examples of participant responses to the SMT. Left an example of Floor (Type b) entry, where the locations are arranged in rows but vertical relationships are not respected (notice that the two locations in the top-row (level 4) are next to each-other although they are in opposite sides of the building. Right an example of Survey (Type c) entry. 


\section{Structure Mapping Task Classification}

The product of the structure mapping task was captured immediately as an image, and all images were classified into four (4) types: (i) floor, (ii) floor x cluster, (iii) survey, and (iv) unclear. These types capture different possibilities for the hierarchical organisation (regionalisation) of multilevel spatial knowledge that might have been adopted by participants: by floor, by vertical cluster (topview/survey), by both floor and vertical cluster, or none (unclear). This scheme has similarities to the one used by Büchner et al. (2007), such as the distinguishing between vertical and horizontal representations, but with a few differences. Representations were not rated on a continuous scale of verticality and horizontality, but each solution was assigned into one of the categories. In addition, because of the building layout, we added a 'survey' type, to capture cases where the targets were arranged from a top view perspective (Figure 5), and we also added an 'unclear' type for ambiguous responses.

Horizontal for cases when the locations were organised in rows corresponding to their floor-level but without making reference to the vertical relationship between locations;

Floor $\mathbf{x}$ Cluster when the locations were organised in rows for each floor, as well as also showed a clear understanding of vertical relationships, e.g. creating a column of overlapping locations;

Survey when the locations were organised in top-view;

Unclear when a strategy was not clearly identifiable.

Errors Errors were noted for each response, by counted all instances were a location was misplaced, according to the participants' own strategy. For example, in the survey mode (top-down view) an error was identified when a target was placed near the wrong group. In the floor-based, an error was identified when a target was placed in a row corresponding to the wrong floor. We did not count errors for the unidentifiable cases.

The images were classified by two independent raters, naive to the purposes and the design of the experiment (Cohen's Kappa for 2 Raters $=0.719, p-$ value $=<0.001$ ) and those cases where the two raters disagreed were resolved by the first author (Fleiss $K$ for 3 raters $=0.79, z=11.7$ ).

\section{M-TSP Performance}

Distance walked During the experiment, the navigation trajectories participants walked to complete the M-TSP tasks were manually recorded on a paper-and-pencil map by the experimenter who was following the participant, and also recorded by a chest-mounted video camera for validation purposes. The trajectories were then digitised in the architectural design software Rhinoceros (Version 5.14, McNeel, 2017). Trajectories were drawn as one polylinefor each TSP transition (i.e. a each subroute connecting one target with the next). The trajectories were traced over the centre-line of all walkable spaces (Figure $2 \mathrm{~B}$ ). Finally, to assess wayfinding performance, we computed the length (i.e. distance walked) of each transition between each two locations, the length of each M-TSP (sum of all transitions), as well as the count of floor-level changes.

To evaluate wayfinding and M-TSP performance, we computed a bi-directional, TSP-transition distance matrix, i.e. the shortest (optimal) path for each pair of locations (origin-destination pair) in the 


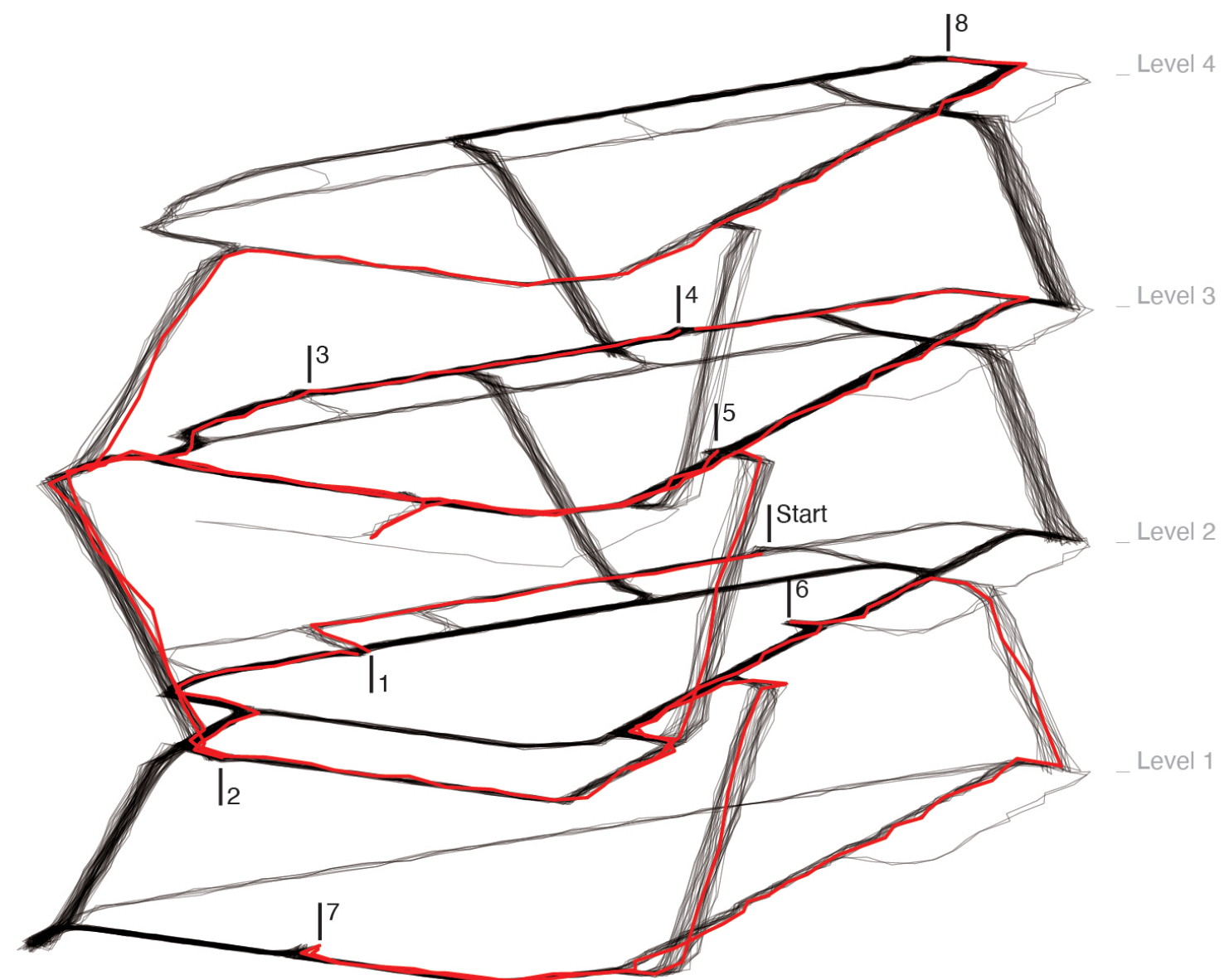

All trajectories / one participant Numbers indicate order of visit

Figure 6: Isometric view of participant trajectories in 3D space, during the two M-TSP tasks. Participant trajectories (black lines) were manually recorded during the task, and later digitised as 3Dpolylines. Red lines present an example of a single TSP path from one participant (Q30, Task 1), targets are indicated with a vertical bar and annotated with the order of visit. Note that in order to improve visual clarity for this figure, the trajectories are plotted here with (i) a horizontal jitter ( \pm 1 meter, in the $x$ and $y$ axes) and (ii) exaggerated vertical distance multiplied by a factor of 5 .

TSP (shopping list) including the starting location. All publicly accessible and navigable spaces of the building were drawn as a spatial (graph) network (Figure $2 \mathrm{~B}$ ), where each corridor of the building is represented as an edge; target locations and intersections were represented as nodes. The distance matrix was bi-directional to account for possible route differences between $A B$ and $B A$, given that escalator edges are directional, going either upwards or downwards. Subsequently, the euclidean distance between all locations was computed as the straight line connecting each OD pair; thus the euclidean distance represents a line that may cross through walls and floors. The shortest (optimal) distance between each two locations was computed using the $A^{*}$ search algorithm, accessed using a custom Grasshopper script (the native scripting environment of Rhinoceros CAD) and the thirdparty component ShortestWalk (https://github.com/mcneeleurope/ShortestWalk; Piacentino, 2011). Finally, for each of the two M-TSP tasks, the optimal solution was identified by a brute-force approach: deriving all 40320 possible permutations, and then computing the path and euclidean distance, as well as the number of floor changes. 
Excess distance walked At a TSP, route optimisation takes place at two levels: first, the route between each successive origin and destination (local optimisation) and, second, the overall sequence of destinations(Hui et al., 2009). For instance, it is possible that a navigator identifies the optimal tour (TSP sequence), but under-performs some of the transitions (i.e. in navigating between the origindestination pairs). Therefore, to evaluate this, participant trajectories were evaluated between each check-point (origin-destination pair). For each participant an ordered list of paths was created: from start, to first target, second target until the last target (task end).

To evaluate wayfinding performance on the TSP tasks, the established measure of percentage above optimal or PAO Wiener et al. (2004) was used, based on the following equation as:

$$
\text { Percent Above Optimal }(P A O)=\frac{\text { distance }- \text { optimal distance }}{\text { optimal distance }} * 100
$$

However, due to the nature of a TSP in a large-scale complex environment, it is possible to identify the optimal sequence (TSP solution), but choose sub-optimal routes locally, i.e. between each successive origin and destination. Based on the definition by (Garling, 1989, see also section* XX above), three performance measures were defined: first the local PAO corresponding to distance travelled above the optimal between each origin-destination pair (i.e. this measure is computed 8 times for every TSP), second the total PAO referring to the distance travelled above the route length of the optimal sequence and, third the global PAO referring to the distance travelled for the realised sequence if each OD was travelled optimally. Indeed, a participant may achieve a very low local PAO (i.e. find the shortest path between an OD pair) and yet have a very high global PAO, if the order of visit to the TSP targets is sub-optimal. Further, we distinguished local PAO (i.e. between consecutive origin-destination pairs) into within-floor and between-floor, to examine whether the number of floorchanges impact wayfinding performance.

Table 2 shows descriptive statistics for participants performance. PAO was computed at two levels: transition-PAO comparing the walked versus the shortest distance between each origindestination pair and the tour-PAO comparing the total walked distance versus total distance of the optimal TSP solution. PAO measures were computed for walked (i.e. path) and for euclidean distance.

Tour sequence optimality We assessed whether the tour was the optimal or how different it was from the optimal. For this measure we only looked on the tour sequence (i.e. order of visiting the targets) without taking into account distance. Each location was assigned a unique letter, such that every M-TSP solution can be represented as a string of letters. To compare between the optimal and the observed tours, we used the levenshtein distance (LV) string difference method. LV compares the number of substitutions necessary to transform one sting to another. For example, taking the stringabcdef as start, and adding 1 change afbcde ( $L V=2), 2$ changes abcdef $(L V=3)$, 3 changes adfbce (LV = 4) and so on. For example, in the case of participant Q05, the optimal TSP sequence for task A was 'adgicejhf', while the observed TSP sequence was 'ajhecigdf', resulting in a LV of 6 (ie. six substitutions). Levenshtein distance was computed using the R-package stringdist (van der Loo, 2014), function stringdist ("abcdef", "adfbce", method = "lv").

Tour PAO One limitation of the levenshtein distance measure is that it disregards the actual distance between targets - tours different from the optimal (ie. LV $i 0$ ) can have only small differences in 
Table 2: Descriptive statistics of M-TSP performance. $\mathrm{N}=41$ participants $\times 2$ tasks.

\begin{tabular}{llll}
\hline Characteristic & Overall $(\mathrm{N}=82)$ & Horizontal $(\mathrm{N}=44)$ & Vertical $(\mathrm{N}=38)$ \\
\hline Mental demand (TLX) & $2.0(-3.0,5.0)$ & $2.5(-3.5,5.0)$ & $1.5(-2.2,5.0)$ \\
Distance walked & $823.4(748.0,931.2)$ & $851.4(754.8,981.1)$ & $814.9(742.2,873.8)$ \\
TSP percentile (path) & $2.0(0.4,5.7)$ & $2.8(0.6,12.8)$ & $1.6(0.3,3.4)$ \\
TSP percentile (euclidean) & $31.4(10.7,53.6)$ & $37.8(13.5,58.5)$ & $22.7(8.5,46.3)$ \\
Excess distance (\%, Overall) & $36.2(26.7,59.7)$ & $44.4(26.8,68.4)$ & $34.8(26.5,50.0)$ \\
Excess distance (\%, tour) & $13.8(7.9,24.0)$ & $14.1(8.4,23.9)$ & $13.8(4.2,24.8)$ \\
Excess distance (\%, transition) & $11.6(7.6,22.4)$ & $11.6(7.7,19.6)$ & $11.8(3.8,23.8)$ \\
Floor changes & & & \\
4 & $21(26 \%)$ & $14(32 \%)$ & $7(18 \%)$ \\
5 & $34(41 \%)$ & $16(36 \%)$ & $18(47 \%)$ \\
6 & $15(18 \%)$ & $7(16 \%)$ & $8(21 \%)$ \\
7 & $7(8.5 \%)$ & $4(9.1 \%)$ & $3(7.9 \%)$ \\
8 & $3(3.7 \%)$ & $2(4.5 \%)$ & $1(2.6 \%)$ \\
9 & $1(1.2 \%)$ & $0(0 \%)$ & $1(2.6 \%)$ \\
10 & $1(1.2 \%)$ & $1(2.3 \%)$ & $0(0 \%)$ \\
\hline
\end{tabular}

distance. We analysed this by comparing the excess distance between the optimal tour and the realised distance. We should emphasise that here we used the optimal distance for each tour, and not the actual distance walked for tour as discussed above.

$$
\text { Tour PAO }{ }_{i}=\frac{\text { optimal length } \text { tour }_{i}}{\text { optimal tour length }}
$$

Tour percentile Another way to asses the performance on a TSP task is to compare how optimal is a tour with respect to all other alternative tours, which wee evaluated with the measure of tour percentile. First we computed the excess distance for each tour (tour PAO, above), and then ranked them from the best $(0 \%)$ to worst $(100 \%)$. In this manner we obtained the percentile of each tour, for instance that a given tour is in the best $10 \%$ of all possible tours. Furthermore, to examine if participants optimised their tours based on path- (walkable) or euclidean- (straight line) distance, we computed a tour percentile with both distance measures and compared them. If participants used path-distance for their decision-making, then they would demonstrate lower path-based tour percentiles, whereas if they used euclidean-distance they would demonstrate lower euclidean-based tour percentiles.

Transition PAO To evaluate local wayfinding, in other words the excess distance walked between each two location visited during a tour (in a tour of $a-b-c$, that would correspond to $a b, b c$ ) we computed the percent above optimal (PAO) of each transition.

$$
\text { Transition } P A O_{i}=\frac{\text { path distance } \text { transition }_{i}}{\text { shortest path distance transition }}
$$

\subsubsection{M-TSP strategies}

The wayfinding strategies used by participants to resolve the TSP will be evaluated from their routes, based on the sequence of visits to the available targets of each task. We will examine two typical 
strategies for solving TSPs: nearest neighbour and regionalisation (clustering).

Nearest neighbour The NN heuristic consists of visiting the immediately nearest target, repeat until completing a tour. Depending on the spatial organisation of targets it may not lead to an optimal tour, but it is considered cognitively easier as it only requires the computation of the most proximal target. Here we will use the M-TSP distance matrix (discussed above) to compute if participants chose the NN at each transition, and if their choices differed when the NN was on a different floor.

Regionalisation The experiment will test how people resolve spatial tasks with competing region and cluster based strategies. We hypothesise that people consider floors as hierarchical regions Wiener \& Mallot (2003b), where locations can be clustered within or between regions. For example, two locations can be proximal in the horizontal plane (within region) or on the vertical plane (between regions / floors). Our hypothesis is that people will prioritise staying within each region until the find all targets, violating proximity or path optimality in the vertical axis.

\subsubsection{Discrete Choice}

Finally, to understand the joint influence of training, distance, floor changes and strategy on the MTSP decision-making, we adopted a discrete choice framework (McFadden, 1978). Discrete choice models have been used in healthcare, marketing and are applied in transport demand modeling, among others, to understand individual's mode choice, route choice, destination choice. Choice models assume a decision-maker chooses an alternative from a set of finite alternatives. An alternative is specified by a series of attributes. Parameters are estimated that describe the level of utility or disutility of each attribute; the sum of utilities and disutilities results in the utility of an alternative, which can be negative or positive. It is assumed, that the alternative with the highest utility is chosen. The decision-maker is a utility maximixing, rational, agent. However, the analyst is assumed to have incomplete information and, therefore, a measurement error must be taken into account. Four sources of uncertainty can be recognized: unobserved alternative attributes unobserved individual characteristics, measurement errors and proxy variables. In order to reflect this fact the error is modelled as a random variable. In the case of pedestrian route choice, common attributes for route alternative include distance, comfort, perceived safety and waiting time at intersections; some of these attributes might be deemed more important to the decision-maker than others. Distance usually is perceived negative for utilitarian trips: decision-makers prefer routes with shorter distances. Comfort might be perceived positive. The results of discrete models allow the analyst to estimate and quantify these trade-offs. In the study at hand, we assume that participants solved the M-TSP sequentially, deciding their next target one by one. Thus, at start they have to choose between 8 alternatives, then 7 and so on, until there is only one target left. In our case we assumed participants considered three main costs: path distance, number of floor changes and whether it is part of a vertical cluster. Additionally we tested the interaction between sense of direction ability and path distance, and the interaction between training and the subjective utility floor changes and vertical clusters.

\section{Statistical analysis}

All statistical analysis were performed in $\mathrm{R}(\mathrm{R}$ Core Team, 2021). We used the package BayesFactor (Morey \& Rouder, 2018) for bayesian contigency tables (equivalent to $\chi^{2}$ tests). We used bayesian 
A

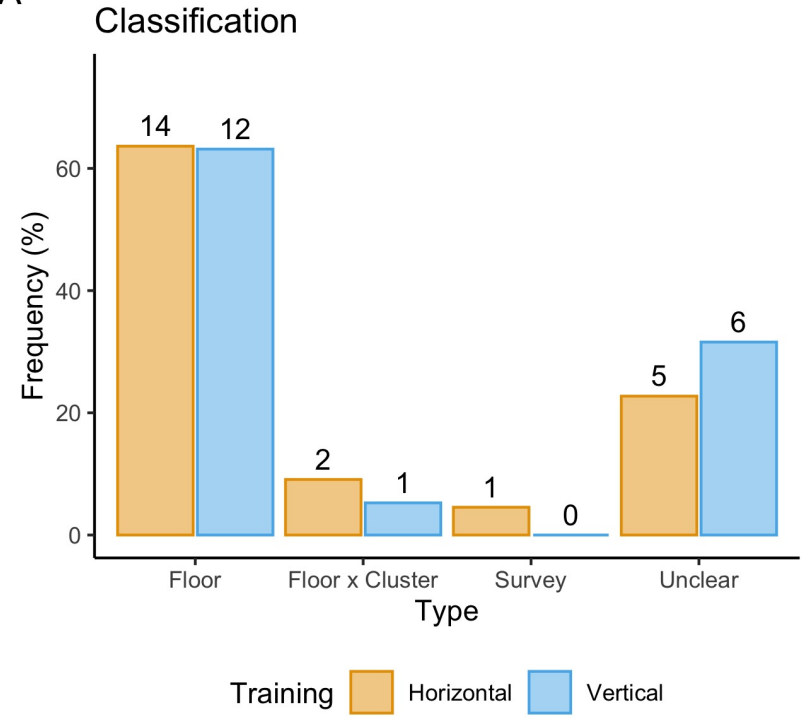

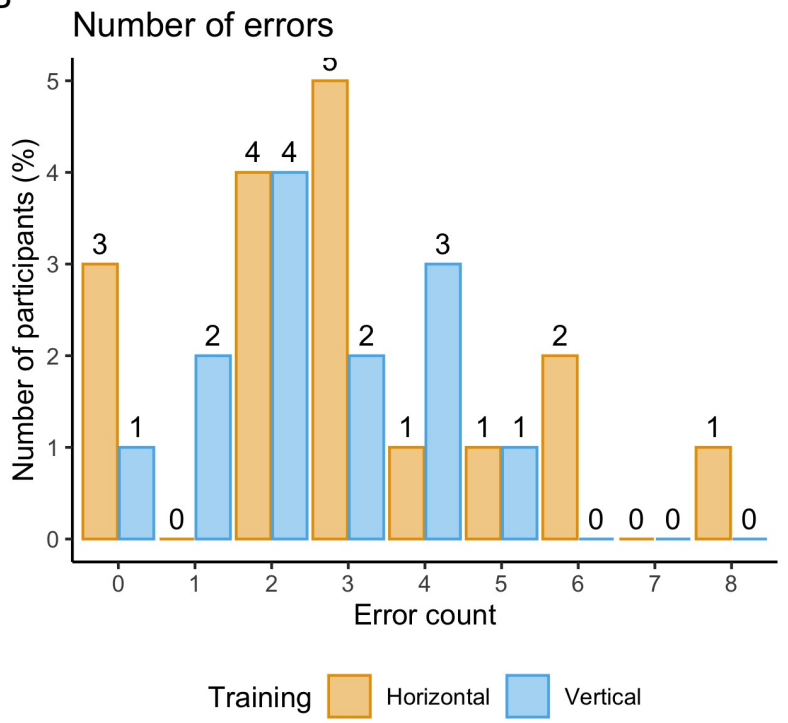

Note that the error count (B) does not include 'unclear' responses from classification (A).

Figure 7: Frequency of 3D spatial representation types at the Structure Mapping Task $(N=41)$.

mixed-effects models implemented in the brms package in $\mathrm{R}$ (Bürkner, 2017). All models were estimated over 4 chains of 4000 iterations, with a burn-in period of 2000 samples, and weakly informative priors centered on zero. Model performance was assessed with graphical posterior predictive checks, that is comparing data simulated from the posterior predictive distribution with the observed data (Gabry et al., 2019). In all models, we entered as fixed effects, we entered training, task, sense of direction (SBSOD, scaled) and familiarity. As random effects, we added intercepts for subjects. We report summary statistics for each parameter using the package bayestestR (Makowski et al., 2019a), which inlude the median, 95\% credible intervals, as well as the probability of direction (pd) which refers to the proportion of the posterior distribution of parameter that is in the same direction (i.e. sign) as the median, and can be interpreted as a directional hypothesis test that the probability that a parameter is strictly positive or negative (Makowski et al., 2019b). Further, to quantify the evidence in favour of this directional hypothesis, we calculated an evidence ratio (Bürkner, 2017) defined as the posterior probability $(p d)$ under the hypothesis against its alternative (i.e. $P(A>B) /$ $P(B>A)$ or $P(A>B) / 1-P(A>B))$. We describe the evidence for a hypothesis, as moderate if $85 \%$ of the posterior is in the same direction,

The discrete choice model (multinomial logit) was estimated using the R-package Apollo (Hess \& Palma, 2019) using maximum likelihood estimation.

\section{Results}

\subsection{Structure Mapping Task}

First we assessed the effect of training (horizontal vs vertical) on 3D spatial representations by analysing the structure mapping task.

Table 3 shows descriptive statistics including the 3D representation type and the number of errors. We used a Bayesian test of association (contingency table) using version 0.9.12-4.2 of the BayesFac- 
Table 3: Frequency of representation type in the structure mapping task

\begin{tabular}{llll}
\hline Category & Horizontal training & Vertical training & Total \\
\hline Rows & 14 & 12 & 26 \\
Rows \& Columns & 2 & 1 & 3 \\
Survey & 1 & 0 & 1 \\
Unclear & 5 & 6 & 11 \\
Total & & & 41 \\
\hline Average Error & $3.16(2.22)$ & $2.54(1.45)$ & \\
\hline
\end{tabular}

Table 4: Parameter estimates for errors in the structure mapping task (11 unclear responses were ommitted, hence number of observations: 30 ).

\begin{tabular}{lrlrrrrr}
\hline Parameter & Median & $95 \% \mathrm{Cl}$ & pd & Rhat & ESS & evid.ratio & * \\
\hline Intercept & -1.052 & $(-1.419,-0.702)$ & 1.00 & 1 & 4203 & Inf & * \\
Training Vertical & -0.254 & $(-0.751,0.248)$ & 0.84 & 1 & 4112 & 5.36 & \\
Familiar & -0.080 & $(-0.570,0.412)$ & 0.63 & 1 & 4211 & 1.68 & \\
SBSOD (sc.) & 0.024 & $(-0.226,0.295)$ & 0.57 & 1 & 4207 & 1.33 & \\
\hline
\end{tabular}

Note: $\quad$ Median; $95 \%$ Credible Intervals; pd: probability of direction; ESS: effective sample size; evid.ratio: evidence ratio; star: posterior probability exceeds $95 \%$.

tor package with default priors and a independent multinomial sampling plan Morey \& Rouder (2018), to investigate the effect of training, spatial ability and familiarity on the representation type (including the unclear category). We found evidence in favour of the null hypothesis, i.e. the independence of representation type and either training $\left(B F_{\text {null }}=14.6\right)$, sense of direction $\left(B F_{\text {null }}=14.6\right)$ or familiarity $\left(B F_{\text {null }}=18.86\right)$. The null effect of training persisted even after excluding unclear responses $\left(B F_{\text {null }}=9.09\right)$. In other words, the horizontal or vertical guided tour did not lead to systematic preferences for a representation type. Since we did not find evidence for an effect of training on representation type, we pooled together the representations types from both groups, to examine if there is systematic preference for a specific type of representation. There were four possible categories of response, so we set the chance level at $25 \%$. The test of association between expected versus observed frequencies, showed decisive evidence in favour of a systematic preference for representation type rows $(B F=438.98)$. Finally, we conducted a bayesian logistic regression to examine whether the number of errors was influenced by training, spatial ability or familiarity. Table 4 shows that there was anecdotal evidence (84\% posterior) for a negative effect (lower errors) of vertical training; the effects of other parameters were inconclusive.

\subsection{M-TSP performance}

In this section we present the results of participant behaviour on the M-TSP tasks. This is described through multiple measures: mental demand, distance walked, tour sequence optimality, tour percentile, excess tour distance, excess transition distance, and also use of the nearest neighbour and regionalisation strategies. For all following analysis, unless specified otherwise, we fitted bayesian mixed effect models, using brms (Bürkner, 2017) entering fixed effects for training, task and sense of direction, and random effects by participant. Note that we refer as path distance to the length of a walkable route, and as euclidean distance the length of the direct line connecting two locations (i.e. 
the euclidean distance as defined here crosses floors and walls).

M-TSP Navigation performance

A

Distance walked

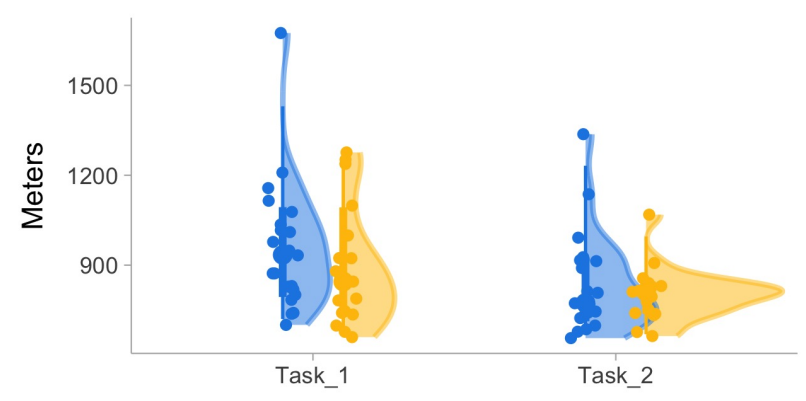

C

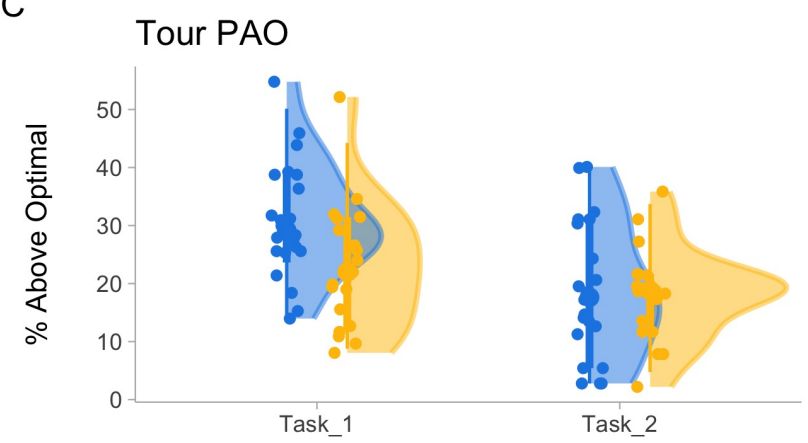

$E$

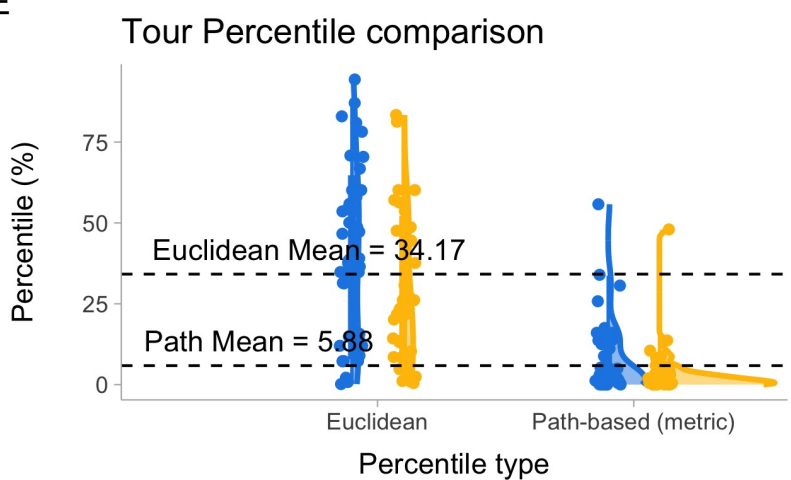

B

\section{Global PAO}

Distance walked versus Optimal Tour Length

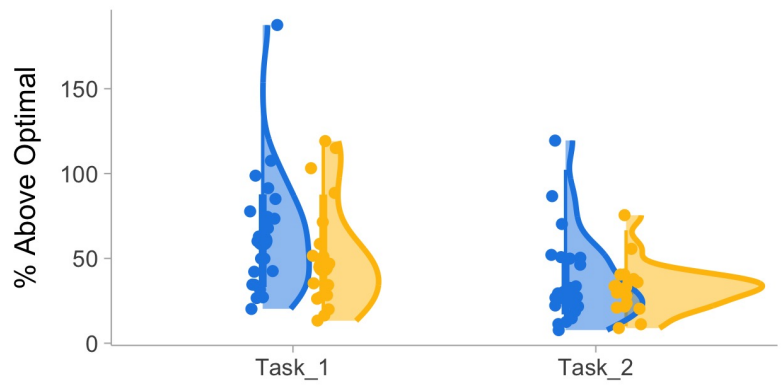

D

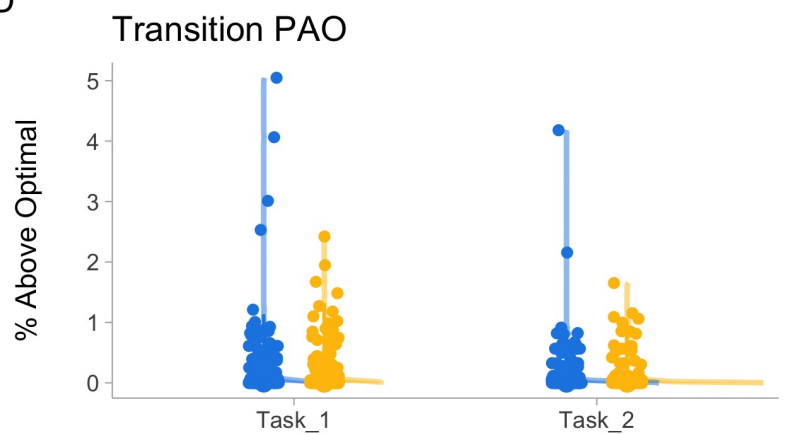

$\mathrm{F}$

Tour sequence optimality

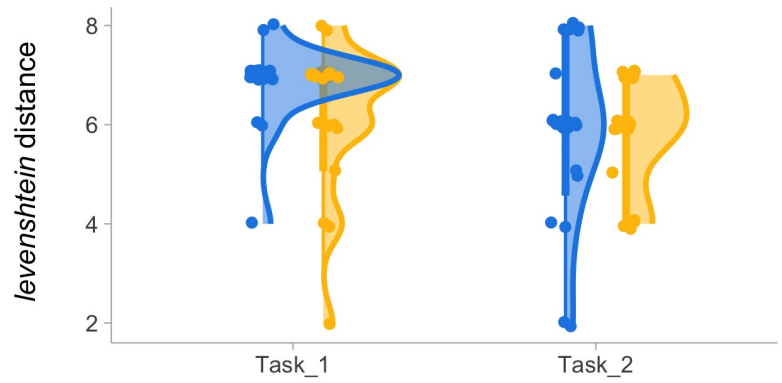

Training

Figure 8: Navigation performance in the MTSP tasks.

Mental Demand Taking into account the ordinal nature of the responses to self-reported mental demand (NASA TLX subscale) we fitted a multilevel ordinal regression Bürkner \& Vuorre (2019) specifying mental demand as the dependent ordinal variable. Table 5 shows the parameter estimates. We found that training (horizontal or vertical) and familiarity appeared to be unrelated to mental demand, but there was substantial evidence that higher sense of direction was associated with lower mental demand ( $88 \%$ of posterior) , and decisive evidence ( $100 \%$ of posterior) that the TSP required less mental demand on the second task. 
Table 5: Parameter estimates for ordinal regression model of self-reported mental demand (NASA TLX). Intercepts are removed for brevity.

\begin{tabular}{lrlrrrrr}
\hline Parameter & Median & $95 \% \mathrm{Cl}$ & pd & Rhat & ESS & evid.ratio & * \\
\hline Training Vertical & 0.386 & $(-1.697,2.549)$ & 0.64 & 1 & 1965 & 1.79 & \\
Task 2 & -1.620 & $(-2.626,-0.701)$ & 1.00 & 1 & 3567 & 2665.67 & * \\
SBSOD (sc.) & -0.647 & $(-1.817,0.446)$ & 0.88 & 1 & 1937 & 7.39 & \\
Familiar & -0.155 & $(-2.350,2.185)$ & 0.55 & 1 & 1932 & 1.24 & \\
\hline
\end{tabular}

Note: $\quad$ Median; 95\% Credible Intervals; pd: probability of direction; ESS: effective sample size; evid.ratio: evidence ratio; star: posterior probability exceeds $95 \%$.

Table 6: Parameter estimates for distance walked.

\begin{tabular}{lrlrrrrr}
\hline Parameter & Median & $95 \% \mathrm{Cl}$ & pd & Rhat & ESS & evid.ratio & * \\
\hline Intercept & 902.275 & $(862.843,944.072)$ & 1.00 & 1 & 13515 & Inf & * \\
Training Vertical & -5.974 & $(-37.440,24.685)$ & 0.65 & 1 & 17286 & 1.84 & \\
Task 2 & -16.978 & $(-47.696,14.599)$ & 0.86 & 1 & 16248 & 5.90 & \\
SBSOD (sc.) & -16.871 & $(-35.984,4.304)$ & 0.95 & 1 & 14399 & 17.75 & \\
Familiar & -20.460 & $(-50.284,12.375)$ & 0.90 & 1 & 16427 & 8.81 & \\
\hline
\end{tabular}

Note: $\quad$ Median; $95 \%$ Credible Intervals; pd: probability of direction; ESS: effective sample size; evid.ratio: evidence ratio; star: posterior probability exceeds $95 \%$.

Distance Walked Overall, the average distance walked was 874 meters, $897 \mathrm{~m}$ for the horizontal group (SD = 197) and $847.9 \mathrm{~m}$ for the vertical group $(S D=156)$. On average, the horizontal group walked more. Table 6 shows the results of a linear mixed effects model of distance walked. There was strong evidence ( $86 \%$ of posterior) that both participants reduced the overall distance walked in the second task, and very strong evidence that participants with higher sense of direction walked less.

Global PAO The overall, or global PAO, calculated as the difference between then distance walked and the length of the optimal tour. On average, the horizontally trained group walked $50.9 \%$ excess distance, whereas the vertically trained group walked $42.3 \%$ in excess of the optimal. Table 6 shows the results of a bayesian mixed model of global PAO (using a skew normal distribution). The effect

Table 7: Parameter estimates for global (overall) PAO.

\begin{tabular}{lrlrrrrr}
\hline Parameter & Median & $95 \% \mathrm{Cl}$ & pd & Rhat & ESS & evid.ratio & * \\
\hline Intercept & 0.582 & $(0.487,0.692)$ & 1.00 & 1 & 5256 & Inf & * \\
Training Vertical & -0.001 & $(-0.091,0.076)$ & 0.51 & 1 & 4934 & 1.06 & \\
Task 2 & -0.140 & $(-0.242,-0.031)$ & 1.00 & 1 & 1694 & 544.45 & * \\
SBSOD (sc.) & -0.028 & $(-0.069,0.014)$ & 0.91 & 1 & 6644 & 10.70 & \\
Familiar & -0.098 & $(-0.189,-0.016)$ & 0.99 & 1 & 5527 & 106.14 & * \\
\hline
\end{tabular}

Note: $\quad$ Median; 95\% Credible Intervals; pd: probability of direction; ESS: effective sample size; evid.ratio: evidence ratio; star: posterior probability exceeds $95 \%$. 
Table 8: Parameter estimates of ordinal regression for tour optimality (levenshtein distance).

\begin{tabular}{|c|c|c|c|c|c|c|c|}
\hline Parameter & Median & $95 \% \mathrm{Cl}$ & $\mathrm{pd}$ & Rhat & ESS & evid.ratio & * \\
\hline Intercept[1] & -8.339 & $(-19.168,-3.860)$ & 1.00 & 1 & 1261 & Inf & * \\
\hline Intercept[2] & -4.651 & $(-6.604,-3.216)$ & 1.00 & 1 & 2707 & Inf & * \\
\hline Intercept[3] & -8.049 & $(-17.993,-3.994)$ & 1.00 & 1 & 1462 & Inf & * \\
\hline Intercept[4] & -3.165 & $(-4.466,-2.088)$ & 1.00 & 1 & 2988 & Inf & * \\
\hline Intercept[5] & -3.796 & $(-5.211,-2.527)$ & 1.00 & 1 & 5369 & Inf & * \\
\hline Intercept[6] & -1.099 & $(-1.902,-0.329)$ & 1.00 & 1 & 9395 & 249.00 & * \\
\hline Intercept[7] & 1.237 & $(0.224,2.364)$ & 1.00 & 1 & 2210 & 234.29 & * \\
\hline Training Vertical & -0.690 & $(-1.506,0.093)$ & 0.96 & 1 & 6232 & 24.81 & * \\
\hline Task 2 & -0.808 & $(-1.634,0.019)$ & 0.98 & 1 & 5707 & 49.96 & * \\
\hline SBSOD (sc.) & 0.208 & $(-0.217,0.616)$ & 0.84 & 1 & 6867 & 5.34 & \\
\hline Familiar & 0.128 & $(-0.668,0.908)$ & 0.62 & 1 & 7636 & 1.66 & \\
\hline
\end{tabular}

Note: $\quad$ Median; $95 \%$ Credible Intervals; pd: probability of direction; ESS: effective sample size; evid.ratio: evidence ratio; star: posterior probability exceeds $95 \%$.

Table 9: Parameter estimates for linear regression model of tour PAO

\begin{tabular}{lrlrrrrr}
\hline Parameter & Median & $95 \% \mathrm{Cl}$ & pd & Rhat & ESS & evid.ratio & * \\
\hline Intercept & 29.156 & $(25.272,33.031)$ & 1.00 & 1 & 8131 & Inf & * \\
Training Vertical & -3.030 & $(-7.247,1.516)$ & 0.91 & 1 & 6827 & 10.38 & \\
Task 2 & -7.349 & $(-11.424,-3.543)$ & 1.00 & 1 & 10115 & 2665.67 & * \\
SBSOD (sc.) & -1.201 & $(-3.651,1.148)$ & 0.84 & 1 & 6200 & 5.07 & \\
Familiar & -3.644 & $(-8.096,0.730)$ & 0.94 & 1 & 7289 & 17.18 & \\
\hline
\end{tabular}

Note: $\quad$ Median; 95\% Credible Intervals; pd: probability of direction; ESS: effective sample size; evid.ratio: evidence ratio; star: posterior probability exceeds $95 \%$.

of training was inconclusive, while there was decisive evidence for a negative effect of task and familiarity. However, the global PAO captures the joint effect of the optimality of the chosen tour as well as the local wayfinding between each two locations. To disentangle this we performed separate analyses described below.

Tour sequence optimality We examined the similarity between optimal tour for each M-TSP task, compared to the tours realised by participants in terms of the order of visiting the locations. To assess this similarity we compared the levenshtein distance (LV) calculated between the optimal and each realised M-TSP tour sequence. Notably, no participant choose the exact optimal sequence, although one participant from each group was very close $(\mathrm{LV}=2)$. The average LV was 6.22 (range = 2 - 8). Table 8 Overall we found very strong evidence that the vertical group produced TSP with a lower LV compared to the horizontal group, and strong evidence that participants chose more optimal tours (i.e. lower LV from the optimal) on the second task.

Tour PAO To understand how the choice of M-TSP tour (visit order) impacted the distance of the tour, we fitted a BLMM on the tour PAO, i.e. the difference between the optimal length of the realised tour (i.e. without wayfinding errors) and the length of the optimal tour. In terms of path distance, the chosen sequences were on average $22 \%$ longer than the optimal sequence (horizontal mean $=$ 
Table 10: Parameter estimates for beta regression model of MTSP percentiles.

\begin{tabular}{lrlrrrrr}
\hline Parameter & Median & $95 \% \mathrm{Cl}$ & pd & Rhat & ESS & evid.ratio & * \\
\hline Intercept & -0.228 & $(-0.862,0.406)$ & 0.77 & 1.00 & 1147 & 3.34 & \\
Percentile Metric & -2.461 & $(-2.733,-2.183)$ & 1.00 & 1.00 & 3319 & Inf & * \\
Training Vertical & -0.703 & $(-1.464,0.034)$ & 0.97 & 1.01 & 1033 & 28.85 & * \\
Task 2 & -0.349 & $(-0.910,0.200)$ & 0.90 & 1.00 & 1299 & 8.85 & \\
Familiar & -0.422 & $(-1.106,0.286)$ & 0.88 & 1.00 & 1441 & 7.54 & \\
SBSOD (sc.) & -0.110 & $(-0.446,0.220)$ & 0.74 & 1.00 & 1798 & 2.89 & \\
\hline
\end{tabular}

Note: $\quad$ Median; 95\% Credible Intervals; pd: probability of direction; ESS: effective sample size; evid.ratio: evidence ratio; star: posterior probability exceeds $95 \%$.

$24.7 \%$; SD $=12.4 \%$; range $=3 \%-55 \%$; vertical mean $=20.5 \%$; $S D=9.8 \%$; range $=2 \%-52 \%$ ). Table 9 shows the results of the BLMM. We found that decisive evidence that participants improved in the second task, strong evidence for a negative effect of vertical training (i.e. lower tour PAO), and very strong evidence that participants previously familiar with the environment chose more optimal tours.

Tour percentiles Given the large number of alternative TSP sequences, many tours may lead to similar overall distance. Accordingly, we examined the TSP sequences in terms of distance percentile. We found that on average, participants' sequences were on the best $2 \%$ among all the alternatives (horizontal training Median $=2.8 \%$; $S D=11.2 \%$; range $=0 \%-56 \%$, versus vertical training Median $=1.6 \%$; SD $=8.0 \%$; range $=0 \%-48 \%$ ).

Because the present study involves a navigational TSP in a real-world building, the straight-line (euclidean) distance between each pair of locations differs from the path (walkable) distance. This allowed us to examine which distance (cost) participants used in their spatial decision making. First, we found a statistically significant positive correlation between the euclidean and the path percentile of each realised tour $(r(80)=.65, p<.001)$. However, as Figure 8 shows, we also noted that the average path-distance percentile (Horizontal group Median $=2.8 \%$; SD $=11.2 \%$; range $=0 \%-56 \%$ \&Vertical group Median $=1.6 \%$; SD $=8.0 \%$; range $=0 \%-48 \%$ ) was a lot lower the average euclidean percentile (Horizontal group Median $=37.8 \%$; SD $=27.0 \%$; range $=0 \%-94 \%$ \& Vertical group Median $=22.7 \%$; SD $=22.9 \%$; range $=0 \%-83 \%$ ). Table 10 shows the results of a bayesian mixedeffects model with a Beta likelihood fitted to percentiles (percentiles are bounded between 0 and 1). We found decisive evidence ( $100 \%$ of posterior) that for the same tour the path-distance (metric) percentile was lower than the euclidean percentile, a well as strong evidence that vertical training led to tours with a lower percentile, and moderate evidence for that familiarity ( $88 \%$ of posterior) and task (90\% of posterior) also resulted in lower TSP percentiles.

Transition PAO We analysed transition PAO, i.e. the distance excess distance walked between any two locations in the tour, which reflects local wayfinding. The horizontal group walked on average $20.5 \%$ more $(S D=21.6 \%$; range $=1 \%-107 \%$ ) whereas the vertical group walked $17.8 \%$ excess distance $(S D=16.8 \%$; range $=0 \%-67 \%)$. Overall, the vertical training group minimised the distance walked (i.e. zero excess distance) during transitions significantly more than the horizontal $\left(\chi^{2}=4.86, d f=1, p\right.$-value $\left.=0.027\right)$; vertical training group minimised the distance walked 
Table 11: Parameter estimates for hurdle gamma regression model of transition PAO

\begin{tabular}{llrlrrrrr}
\hline Part & Parameter & Median & $95 \% \mathrm{Cl}$ & pd & Rhat & ESS & evid.ratio & * \\
\hline & Intercept & -0.981 & $(-1.571,-0.419)$ & 1.00 & 1 & 4829 & 2665.67 & $*$ \\
& Training Vertical & 0.003 & $(-0.512,0.536)$ & 0.50 & 1 & 5921 & 1.02 & \\
Gamma part & Within floor & -0.094 & $(-0.906,0.742)$ & 0.59 & 1 & 4107 & 1.45 & \\
& Vertical cluster & -1.386 & $(-2.381,-0.396)$ & 1.00 & 1 & 4339 & 284.71 & $*$ \\
& Familiar & -0.169 & $(-0.712,0.329)$ & 0.74 & 1 & 6184 & 2.82 & \\
& Task 2 & -0.264 & $(-0.580,0.048)$ & 0.95 & 1 & 12202 & 18.09 & \\
\hline \multirow{5}{*}{ Hurdle part } & Intercept & -1.445 & $(-1.864,-1.055)$ & 1.00 & 1 & 15562 & Inf & $*$ \\
& Training Vertical & 0.408 & $(0.036,0.760)$ & 0.99 & 1 & 18399 & 80.63 & $*$ \\
& Within floor & 2.552 & $(2.122,2.980)$ & 1.00 & 1 & 12170 & Inf & $*$ \\
& Task 2 & -0.208 & $(-0.544,0.159)$ & 0.87 & 1 & 18927 & 6.70 & \\
& Vertical cluster & 0.914 & $(0.454,1.414)$ & 1.00 & 1 & 13227 & Inf & * \\
\hline
\end{tabular}

Note: $\quad$ Median; 95\% Credible Intervals; pd: probability of direction; ESS: effective sample size; evid.ratio: evidence ratio; star: posterior probability exceeds $95 \%$.

$48.4 \%$ of the time (147 out of 304 transitions), while the horizontal group $39.5 \%$ of the time (139 out of 352 transitions). Due to the high number of perfect transitions (i.e. zeroes) we fitted a hurdle regression, specifying a Gamma likelihood for the non-hurdle part. Transition PAO was specified as the outcome variable, entering fixed effects for training, task, spatial ability, familiarity and whether the transition was to the same floor (within floor) or in the same vertical cluster; to account for the repeated measurements, we specified random effects by participant.

Table 11 shows the parameter estimates for transition pao. Starting from the hurdle part, we found evidence that vertical training, transition to a within-floor location or a vertical cluster, all increased the likelihood of zero PAO. In the gamma component of the model we found evidence that navigating to a vertical cluster was associated with lower PAO, and the transition PAO reduced in the second task.

\section{M-TSP strategies}

Nearest neighbour In a TSP task, choosing the nearest neighbour (NN) at each step is presumed to be a cognitively easy solution, but it does not always lead to the shortest travel distance overall Saisa \& Garling (1987). Here we defined the NN based on the shortest path (walkable) distance among the alternatives at each step of the TSP. Overall, both groups chose the NN alternative equally often, specifically $53 \%$ of the time, making in total 302 NN transitions over 574 decisions, 162/308 for the horizontal and 140/266 for the vertical group). There was inconclusive evidence that the vertical group made more transitions to the nearest neighboor on the same floor (horizontal cluster; $b=$ $0.036[-0.514,0.567]$, post. prob $=0.54$, evid.ratio $=1.15$ ), and the the horizontal group made more transitions to the nearest neighbour on a vertical cluster $(b=0.129[-0.519,0.772]$, post.prob $=0.64$, evid.ratio $=1.76$ ).

We then examined those cases when participants did not chose for the nearest neighbour (not$N N$ ), to explore whether groups were biased with respect to floors or vertical clusters (Figure 9). We found that both groups were more likely to chose the NN when it was on the same floor, than on a vertical-region or elsewhere. However, when they made a transition to a non-NN target, we found substantial evidence that the horizontal group were more likely to choose a target on the same floor 
A

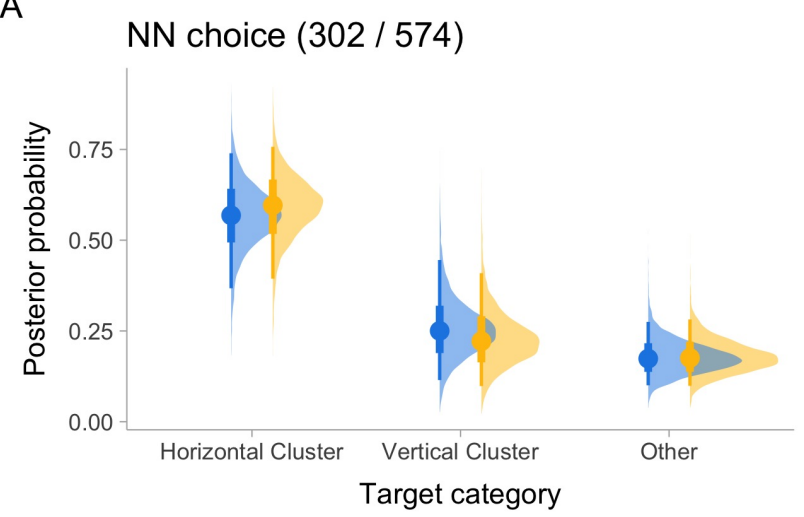

C

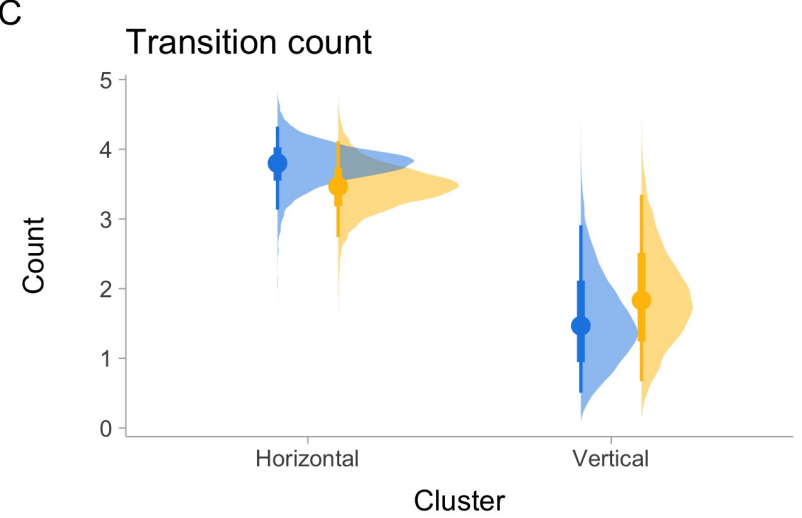

B

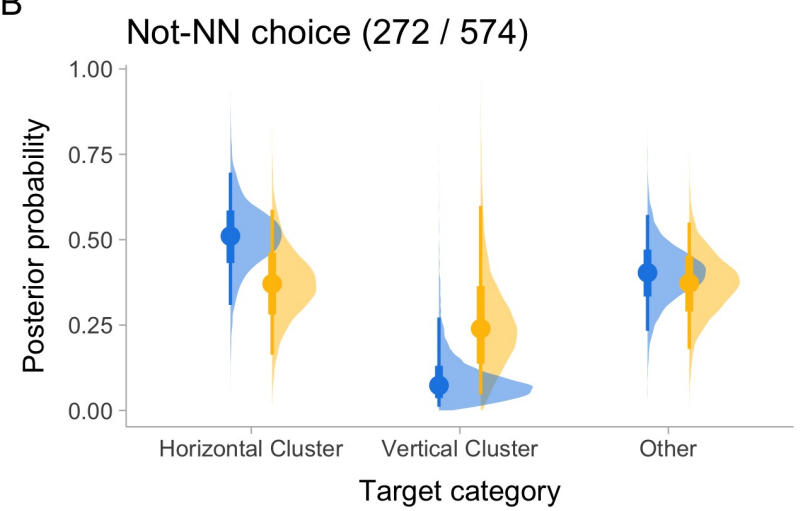

Figure 9: MTSP decision making.

$(H>V, b=0.244[-0.237,0.729]$, post.prob $=0.79$, evid.ratio $=3.87)$, whereas we found decisive evidence that the vertical group were more likely to choose a target on a vertical cluster $(\mathrm{V}>\mathrm{H}, \mathrm{b}=$ $1.271[0.521,2.064]$, post.prob $=1.00$, evid.ratio $=443.44$ ).

Regionalisation To assess the effects of vertical versus horizontal regionalisation, we analysed how often participants chose to navigate to a location within-vertical or within-horizontal region (Figure $9 \mathrm{C}$ ). Based on the distribution of targets across floor and vertical clusters, there are a maximum possible of five (5) within-vertical region choices, and five (5) within-horizontal region (i.e. floor) choices (see Methods). We performed hierarchical mixed-effects binomial regression estimating the number of region choices, specifying training and task as fixed effects, and by-participants. We found that the vertical group was less likely than the horizontal group to make horizontal region (within-floor) choices $(b=-0.336[-0.707,0.043]$, post.prob $=0.93$, evid.ratio $=12.65)$. For the vertically trained group, the odds of choosing a horizontal region during the TSP is decreased by $\exp (-0.336)=0.71$ times, or $30 \%$ lower. In contrast, the odds of choosing a vertical region during the TSP was higher for the vertically trained group $(b=0.335[-0.124,0.802]$, post.prob $=0.88$, evid.ratio $=7.66)$, or approximately $40 \%$ more $(\exp (0.335)=1.40$ times $)$. 
Table 12: Results for Multinomial Logit Model

\begin{tabular}{lrrr}
\hline Parameters & Estimate & Rob. SE & Rob.t-ratio \\
\hline Path Length & -0.017 & 0.002 & -7.196 \\
Floor Changes & -1.536 & 0.164 & -9.383 \\
Vertical Strategy & 0.583 & 0.145 & 4.018 \\
Vertical Training x Floor Changes & 0.072 & 0.244 & 0.294 \\
Vertical Training x Vertical Strategy & 0.543 & 0.281 & 1.930 \\
N Ind & 41.000 & & \\
N Obs & 574.000 & & \\
LL Null & -869.577 & & \\
LL Final & -607.061 & & \\
R2 & 0.302 & & \\
Adj.r2 & 0.296 & & \\
AIC & 1224.122 & & \\
\hline
\end{tabular}

\section{Multinomial Logit Model}

Table 12 shows the results of the multinomial logit model. The results show that as expected path length and the number of floor changes have have a negative influence on subjective utility, while being on the same vertical cluster as the origin has a positive influence. The interaction term shows that the vertical training strengthens the influence of the vertical cluster. Finally, the trade-offs between the variables of length and floor changes were computed showing that 1 level change is perceived as equivalent to 90 meters (i.e. $b_{\text {Floor changes }} / b_{\text {Route Length }}=-1.536 /-0.017$ ).

\section{Discussion}

This study investigated human cognition and behaviour of multilevel environments and volumetric navigation. We focused specifically on how people form spatial representations of multilevel environments, such as buildings, and how they solve multilevel travelling salesperson problems (M-TSP). While they are intrinsic to and familiar part of everyday navigation, M-TSPs have been understudied, although they present researchers and navigators alike with multiple challenges. We hypothesised that regionalisation of mental representations (Wiener \& Mallot, 2003a) would extend in the vertical domain (Büchner et al., 2007). In other words: people learn spaces flexibly by recognising horizontal or vertical regions and that this is reflected both in their (externalised) mental representations of space as well as their navigational behaviour in the M-TSP.

To investigate these, 41 participants learned twelve (12) different target locations (shops) spanning four-levels of a large shopping mall. Subsequently they completed a structure mapping task to assess their 3D mental representation of space, and completed two open, multilevel TSP tasks with 8 targets (9 points including start) spanning four floor-levels. We chose Singapore as a location for such a study, due to the large-number of multilevel environments (shopping centres), which are embedded in everyday life as destinations for work, leisure, shopping, residence or transport and are integrated within buildings and between buildings on multiple levels. Consequently, there is a variety of environments to conduct real-world studies and, accordingly, potential participants are accustomed to multilevel navigation. Below we discuss mental representations of 3D space, multilevel navigation 
and M-TSPs and the influence of training.

Mental representations of volumetric space The 3D mental representations of space were assessed using a structure mapping task, where participants position small cards, representing each target location on a 2D board. Participants from both groups predominantly ( $87 \%$ excluding unclear responses) structured their mental representations favouring regionalisation 'by floor', disregarding spatial relationships in the vertical axis. In addition, we did not find evidence for an effect of training, as participants from both groups were equally likely to produce floor-based representations. Did participants find it difficult to understand the vertical spatial relationships, or were they constrained by the representational medium (2D board)? Three participants from the present study were able to use a dual (rows $x$ columns) strategy to represent the locations, suggesting that it was a feasible solution to the task. A similar approach was used by Büchner et al. (2007) who found that twice as many participants produced vertical (column) than horizontal representations, but also did not find a systematic effect of learning direction. Accordingly, this result points to the difficulty of establishing vertical spatial relationships, inline with earlier findings by Montello \& Pick (1993) who found that participants were slower in pointing and made larger pointing error between floors. In our experiment this was observed despite that several locations pairs were directly co-visible from each other. This finding differs from Büchner et al. (2007) who found that individuals regionalised by vertical connectors (stairs) more often. This difference could be explained by the fact that in our study the locations were positioned in-between escalators, rather than on the escalator, and as a result the spatial relationship was more abstract and directly associated with a spatial feature (i.e. as in Büchner et al). Further, the relatively low occurrence of survey-based (top-view) representations is in line with the argument that spatial learning from experience favours the formation of route rather than survey knowledge (Meilinger et al., 2013). An alternative explanation is that there is a difficulty associated with externalising representations of 3D space on a 2D space medium. Overall this results supports the theory of anisotropy of organisation of cognitive maps (Brandt et al., 2015; Jedidi-Ayoub et al., 2021) and the theory of horizontal bias (Jovalekic et al., 2011).

M-TSP navigation One fundamental aim of cognitive mapping is to enable purposive navigational behaviour (Downs \& Stea, 1973), so beyond the mental representation of volumetric space, we also examined how people resolve a demanding navigational task in a multilevel environment. Following the completion of the structure mapping task discussed above, participants completed 2, 8-target, open, M-TSP navigation tasks, by physically walking to visit each target across the four levels of the building. Unlike single destination wayfinding tasks, the M-TSP enables us to study the anisotropy of 3D movement, by observing horizontal bias, whether individuals prioritise movement in the horizontal rather than the vertical domain.

Overall we found that performance was worse than other types of TSPs, as participants' tour had a global PAO of $47 \%$, i.e. percentage above of optimal distance travelled for the realised sequence if each OD was travelled optimal. Participants tours also deviated substantially from the optimal sequence in terms of order of visiting locations (average levenshtein distance of 6.2). This is in contrast to previous studies reporting very low PAO scores and nearly perfect tour sequence. However, given that our experiment comprised a navigational-TSP, where excess distance is cumulative both from the visit sequence and the wayfinding between targets, we examined these separately. Without considering the wayfinding transitions, participants chose M-TSP tours that in general minimised 
distance (average tour-PAO of $22 \%$ ), performance comparable with Wiener et al's experiment 2 that required participants to navigate using their long-term memory, resulting in a PAO of $17-20 \%$ for $8-9$ point TSPs (Wiener et al., 2009). Transition-PAO, defined as the excess wayfinding distance between an origin and destination was on average 19\%. The dissociation between the sequence error and the wayfinding error are in line with the theory of hierarchical planning(Hayes-Roth \& Hayes-Roth, 1979), and support the idea that the total excess distance in a navigational-TSP can be attributed to two-level hierarchical process, one layer dealing with finding an optimal sequence (tour-PAO) and another layer dealing with local wayfinding (i.e. going from one target to the next; measured with transition-PAO). A separate question regarding navigational TSPs concerns whether people optimise their tours based on path or euclidean distance. Compared with all possible alternatives, participants' tours were on average on the optimal $5.9 \%$ of all M-TSP solutions in terms of path distance, but $34 \%$ in terms of euclidean-paths. The much lower path-distance PAO, suggests that participants heuristic was to minimise path distance, rather than euclidean distance.

We then considered the possible heuristics used to solve the M-TSP tasks. The nearest neighbour algorithm typically provides a simple, although not necessarily optimal, solution to a TSP. In our study we found that participants employed the nearest neighbour strategy equally frequently among both groups (53\% of choices), and there were no differences between groups when visiting a nearestneighbour in a vertical, horizontal region or crossing regions. Notably, when participants did not chose the nearest neighbour, participants from each group showed evidence for a bias towards the regionalisation of their training; participants from the horizontal training group opted for a non-NN target in a horizontal region, whereas participants from the vertical training group opted for a non-NN target in vertical region.

Training Finally, in this study we varied how participants learned the building, 'emphasising' either horizontal or vertical movement. Although we did not find a difference in the externalised mental representations of space (structure mapping task), the behavioural results suggest some small difference. The vertical training group had slightly better TSP and navigation performance across all measures. Further we found that in cases where there were two or more alternatives within up to $25 \%$ excess distance from the nearest neighbour, participants from the vertical group where $10 \%$ more likely to chose the alternative in the vertical cluster, compared to the nearest neighbour. The region-based planning strategy posits that navigators first plan a 'coarse' path to a region and then refine it by visiting nearby locations. Wiener et al. (2009) had found that in a TSP participants try to minimise region crossings. In the present experiment, the TSP tasks included two different types of regions: horizontal (each floor being a region) and vertical (locations overlapping across floors), and we hypothesised that the initial order of visiting the locations during the learning phase would influence how participants organise the targets into regions. We found that while participants from the horizontal training group had approximately higher likelihood of choosing horizontal clusters, participants from the vertical training group had higher likelihood of choosing targets on a vertical cluster. Additionally, the discrete choice model also suggests that vertical training increased preference for vertical transitions (floor changes) and vertical clusters. Conceptually, this is inline with previous studies showing that people are sensitive to how locations are clustered and navigate trying to minimise transitions between clusters (MacGregor, 2015; Wiener \& Mallot, 2003b). 
Synthesis To the best of our knowledge this is the first study to report human TSP behaviour in a multilevel environment such as a building. Taken together, our converging results from the structure mapping task as well as from the M-TSP task provide new behavioural evidence for a horizontal bias in volumetric spatial cognition and behaviour. This is in line with the literature from animal research (Grieves et al., 2021; Jedidi-Ayoub et al., 2021; Jovalekic et al., 2011). We have also shown that this bias can to some extend be attenuated by vertical training, in other words the way a person encounters a multilevel environment. However, despite that training impacted navigational performance and preferences, it did not influence responses to the structure mapping task. This could be explained by a strong effect of floor-level as a spatial hierarchy that people are extensively familiar from everyday life. Alternatively, it could be that the structure mapping task is an inadequate method to capture spatial relationships in volumetric space that extends in all dimensions (three-axes, e.g. non-linear buildings).

Overall, we found a remarkable ability to optimise even in environmental scale space, and also adaptive behaviour, evident by our result that people can resolve TSPs according to the distance metric most suitable to the task, switching from euclidean distance in visual-TSPs (MacGregor, 2017) to considering path distance in the present experiment, i.e. in navigational TSP in large-scale and multilevel environment.

As noted by Widdowson \& Wang (2022) there is an open debate whether spatial representations follow Euclidean principles, what is called the 'euclidean hypothesis'. In their study, they show that people on a 3D pointing task adopt a euclidean-based response, regardless of the curvature properties of the environment (Widdowson \& Wang, 2022). Previous studies have showed that people have the ability to accomplish a 3D pointing task (Brandt et al., 2015; Zwergal et al., 2016) (albeit with evidence of anisotropy). Importantly, in the recent studies of Kim \& Doeller (2021) and Widdowson \& Wang (2022) participants moved along euclidean and non-euclidean spaces defined by their curvature. It has also been shown that people maintain a dual representation, where they keep track of distance both in terms of euclidean and path-distance to calculate detours (Howard et al., 2014). However previous studies have not clarified whether people use euclidean- or path-based information for navigation in 3D space. In our approach participants learned and then navigated physically in a real multilevel building, where space follows euclidean-principles but the valuation of alternative routes can rely on straight-line (euclidean) or path-based distance. Our results show clearly that people can flexibly used their mental representation using a path-based metric of distance when this is ecologically relevant - as in a navigation task.

\subsection{Limitations and future work}

The study presents a number of limitations. First, the specifics of the building layout, including the shape, topological connections between spaces and inter-visibility of locations within and between floors, appear to have influenced the cognition and behaviour of participants. With regards to the structure mapping task, as mentioned above, the two-dimensional method of response may have limited participants in externalising effectively their true mental representations. Future work could investigate other methods, possible using physical or dynamic/digital representations to support response in three-dimensional spatial relationships. With regards to the TSP, the distribution of the target-locations to visit across the four floors favoured horizontal strategies, which may have limited the amplitude of differences between the two groups. Also, the difficulty of the TSP was kept constant, as both tasks required visiting 8 targets. This allowed us to observe wayfinding improvements from 
the first to the second task, but limit our current understanding of navigation in easier or more difficult multilevel TSP. Last, in our paradigm, vertical movement relied on automatic escalators which may have reduced the perceived cost of vertical movement associated with physical effort. Future work can address these limitations by conducting research in other multilevel layouts, varying the difficulty of multilevel TSPs and exploring different types of spatial clustering of locations to understand how regionalisation emerges.

\section{Conclusion}

In this study we investigated how people form mental representations of a multilevel building and how they resolve complex multilevel TSP. Participants learned twelve target locations following a 'horizontal' or 'vertical' type of training. Overall, we found evidence of a vertical anisotropy and horizontal bias both in the structure mapping task and in the M-TSP tasks. Specifically we found that despite the cognitive difficulty of a TSP, participants from both groups achieved highly optimal performance, minimising the overall distance travelled. We observed that the overall deviation from the optimal TSP is a the joint result of sub-optimal choice of TSP solution (accounting for $20 \%$ of excess distance, on average) and sub-optimal performance in navigating between consecutive locations (accounting for an additional $20 \%$ of excess distance, on average). In the analysis of the TSP sequences, we found evidence that the vertical training group increased the probability of vertical movement. These results have implications about our understanding and simulation of human movement in complex buildings. It is important that predictive models of human movement in cities and buildings adequately capture the reluctance for vertical movement. Finally, the TSP and its M-TSP variant provide a powerful framework to study spatial cognition and decision-making in a complex environments.

\section{Acknowledgements}

We are grateful to the management of Westgate Shopping Mall, Singapore, for granting us permission to conduct this study in their premises. We would also like to thank Dr Victor Schinazi and Dr Tyler Thrash for their suggestions in the study design, Linda Wei and Jan Nussbamer for their assistance with data collection, as well as Dr Jakub Krukar for his advice on bayesian statistical methods.

\section{Funding}

The research was conducted at the Future Cities Lab at the Singapore-ETH Centre, which was established collaboratively between ETH Zurich and the National Research Foundation Singapore. This research is supported by the National Research Foundation, Prime Minister's Office, Singapore under its Campus for Research Excellence and Technological Enterprise (CREATE) programme.

\section{Declarations of interest}

None. 


\section{A Appendix}

The locations for the structure mapping and the M-TSP tasks (i.e. the shopping list) consisted of the following locations 12 (8 used as TSP targets). Because the store names are unrelated with the purpose of the study and may have changed since the experiment was conducted, we provide a list in terms of the retail floor-plan, in a format commonly recognisable in Singapore: \#floor-unit: \#01-09, \#01-26, \#02-02,\#02-18, \#02-30, \#02-40, \#03-20,\#03-24, \#03-38, \#03-50 , \#04-05, \#04-36 (TSP targets underlined).

\section{References}

Agrawal, Asha Weinstein, Schlossberg, Marc, \& Irvin, Katja. 2008. How far, by which route and why? A spatial analysis of pedestrian preference. Journal of Urban Design, 13(1), 81-98.

Anderson, D. John. 1983. Optimal foraging and the traveling salesman. Theoretical Population Biology, 24(2), $145-159$

Blaser, R. E., \& Wilber, Julie. 2013. A comparison of human performance in figural and navigational versions of the traveling salesman problem. Psychological Research, 77(6), 761-772.

Blauvelt, Andrew, Yoos, Jennifer, \& James, Vincent. 2016. Parallel Cities: The Multilevel Metropolis. Walker Art Center.

Borgers, Aloys, \& Timmermans, Harry. 2014. Indices of Pedestrian Behavior in Shopping Areas. Procedia Environmental Sciences, 22, 366-379.

Brandt, Thomas, Huber, Markus, Schramm, Hannah, Kugler, Günter, Dieterich, Marianne, \& Glasauer, Stefan. 2015. "Taller and shorter": Human 3-D spatial memory distorts familiar multilevel buildings. PLOS ONE, 10(10).

Büchner, Simon J, Hölscher, Christoph, Wiener, Jan M, Büchner, Simon J, Hölscher, Christoph, \& Wiener, Jan M. 2009. Search Strategies and their Success in a Virtual Maze. Proceedings of the Annual Meeting of the Cognitive Science Society, 31(31), 1066-1071.

Büchner, S.J., Hölscher, Christoph, Strube, G., Büchner, S.J., Buechner, S, Hölscher, Christoph, Strube, G., \& Büchner, S.J. 2007. Path choice heuristics for navigation related to mental representations of a building. Proceedings of the Euro CogSci 2007, 504-509.

Bürkner, Paul Christian. 2017. brms: An R package for Bayesian multilevel models using Stan. Journal of Statistical Software, 80(1).

Bürkner, Paul-Christian, \& Vuorre, Matti. 2019. Ordinal Regression Models in Psychology: A Tutorial. Advances in Methods and Practices in Psychological Science, 2(1), 77-101.

Chang, Dongkuk. 2002. Spatial choice and preference in multilevel movement networks. Environment and Behavior, 34(5), 582-615.

Christofides, Nikos. 1975. HAMILTONIAN CIRCUITS AND THE TRAVELLING SALESMAN PROBLEM. Pages 149-171 of: Roy, B (ed), Combinatorial Programming: Methods and Applications. Dordrecht.Holiand: D. Reidel Publishing Company.

Chronicle, Edward P., MacGregor, James N., Ormerod, Thomas C., \& Burr, Alistair. 2006. It looks easy! Heuristics for combinatorial optimization problems. Quarterly Journal of Experimental Psychology, 59(4), 783-800.

Cook, William J. 2011. In Pursuit of the Traveling Salesman: Mathematics at the Limits of Computation. Princeton University Press.

Cramer, Audrey E., \& Gallistel, C.R. 1997. Vervet monkey as travelling salesman. Nature, 387, 464.

Davis, Victoria A., Holbrook, Robert I., \& Burt de Perera, Theresa. 2018. The influence of locomotory style on three-dimensional spatial learning. Animal Behaviour, 142, 39-47.

Dollé, Laurent, Droulez, Jacques, Bennequin, Daniel, Berthoz, Alain, \& Thibault, Guillaume. 2015. How the learning path and the very structure of a multifloored environment influence human spatial memory. $A d$ vances in Cognitive Psychology, 11(4), 156-162. 
Downs, Roger M, \& Stea, David. 1973. Cognitive maps and spatial behavior: Process and products. Image and Environment, 8-26.

Feng, Yan, Duives, Dorine, \& Hoogendoorn, Serge. 2022. Wayfinding behaviour in a multi-level building : a comparative study of HMD VR and Desktop VR. Advanced Engineering Informatics, 51(November 2021), $1-27$.

Gabry, Jonah, Simpson, Daniel, Vehtari, Aki, Betancourt, Michael, \& Gelman, Andrew. 2019. Visualization in Bayesian workflow. Journal of the Royal Statistical Society. Series A: Statistics in Society, 182(2), 389-402.

Gärling, T, \& Gärling, E. 1988. Distance minimization in downtown pedestrian shopping. Environment and Planning A, 20(4), 547-554.

Garling, Tommy. 1989. The role of cognitive maps in spatial decisions. Journal of Environmental Psychology, 9(4), 269-278.

Gath-Morad, Michal, Thrash, Tyler, Schicker, Julia, Hölscher, Christoph, Helbing, Dirk, \& Aguilar Melgar, Leonel Enrique. 2021. Visibility matters during wayfinding in the vertical. Scientific Reports, 11(1), 115.

Golledge, Reginald. 1999. Human Wayfinding and Cognitive Maps. In: Wayfinding Behaviour: Cognitve Mapping and Other Spatial Processes. Baltimore and London: The Johns Hopkins University Press.

Grieves, Roddy M., Jedidi-Ayoub, Selim, Mishchanchuk, Karyna, Liu, Anyi, Renaudineau, Sophie, Duvelle, Éléonore, \& Jeffery, Kate J. 2021. Irregular distribution of grid cell firing fields in rats exploring a 3D volumetric space. Nature Neuroscience, 24(11), 1567-1573.

Grobéty, Marie Claude, \& Schenk, Françoise. 1992. Spatial learning in a three-dimensional maze. Animal Behaviour, 43(6), 1011-1020.

Guo, Zhan. 2009. Does the pedestrian environment affect the utility of walking ? A case of path choice in downtown Boston. Transportation Research Part D, 14(5), 343-352.

Hales, Jena B., Petty, Elizabeth A., Collins, Gequasha, \& Blaser, R. E. 2021. Contribution of the hippocampus to performance on the traveling salesperson problem in rats. Behavioural Brain Research, 405(February).

Hanyu, K, \& Itsukushima, Y. 2000. Cognitive distance of stairways: a multi-stairway investigation. Scandinavian journal of psychology, 41(1), 63-9.

Hart, Sandra G. 2006. NASA-task load index (NASA-TLX); 20 years later. Proceedings of the Human Factors and Ergonomics Society, 904-908.

Hart, Sandra G, \& Staveland, Lowell E. 1988. Development of NASA-TLX (Task Load Index): Results of Empirical adn Theoretical Research. Advances in Psychology, 52, 139-183.

Haxhimusa, YIl, Carpenter, Edward, Catrambone, Joseph, Foldes, David, Stefanov, Emil, Arns, Laura, \& Pizlo, Zygmunt. 2011. 2D and 3D Traveling Salesman Problem. The Journal of Problem Solving, 3(2).

Hayes-Roth, Barbara, \& Hayes-Roth, Frederick. 1979. A cognitive model of planning. Cognitive Science, 3(4), 275-310.

Hegarty, Mary, Richardson, AE, \& Montello, DR. 2002. Development of a self-report measure of environmental spatial ability. Intelligence, 30, 425-447.

Hess, Stephane, \& Palma, David. 2019. Apollo: A flexible, powerful and customisable freeware package for choice model estimation and application. Journal of Choice Modelling, 32.

Hirtle, Stephen C., \& Garling, Tommy. 1992. Heuristic rules for sequential spatial decisions. Geoforum, 23(2), 227-238.

Hölscher, Christoph, Meilinger, Tobias, Vrachliotis, Georg, Brösamle, Martin, \& Knauff, Markus. 2006. Up the down staircase: Wayfinding strategies in multi-level buildings. Journal of Environmental Psychology, 26(4), 284-299.

Hölscher, Christoph, Büchner, Simon J., Meilinger, Tobias, \& Strube, Gerhard. 2009. Adaptivity of wayfinding strategies in a multi-building ensemble: The effects of spatial structure, task requirements, and metric information. Journal of Environmental Psychology, 29(2), 208-219.

Howard, Lorelei R, Javadi, Amir Homayoun, Yu, Yichao, Mill, Ravi D, Morrison, Laura C, Knight, Rebecca, Loftus, Michelle M, Staskute, Laura, \& Spiers, Hugo J. 2014. The hippocampus and entorhinal cortex encode the path and Euclidean distances to goals during navigation. Current biology : $C B, 24(12), 1331-$ 40. 
Hui, Sam K., Fader, Peter S., \& Bradlow, Eric T. 2009. The traveling salesman goes shopping: The systematic deviations of grocery paths from TSP optimality. Marketing Science, 28(3), 566-572.

Jedidi-Ayoub, Selim, Mishchanchuk, Karyna, Liu, Anyi, Renaudineau, Sophie, Duvelle, Éléonore, \& Grieves, Roddy M. 2021. Volumetric spatial behaviour in rats reveals the anisotropic organisation of navigation. Vol. 24. Springer Berlin Heidelberg.

Jeffery, Kathryn J., Jovalekic, Aleksandar, Verriotis, Madeleine, \& Hayman, Robin. 2013. Navigating in a threedimensional world. Behavioral and Brain Sciences, 36(05), 523-543.

Jovalekic, Aleksandar, Hayman, Robin, Becares, Natalia, Reid, Harry, Thomas, George, Wilson, Jonathan, \& Jeffery, Kate. 2011. Horizontal biases in rats' use of three-dimensional space. Behavioural Brain Research, 222(2), 279-288.

Kim, Misun, \& Doeller, Christian F. 2021. Adaptive cognitive maps for curved surfaces in the 3D world. bioRxiv, 2021.08.30.458179.

Kirsch, Alexandra. 2012. Hierarchical Knowledge for Heuristic Problem Solving - A Case Study on the Traveling Salesperson Problem. Pages 87-102 of: First Annual Conference pn Advances in Cognitive Systems.

Krukar, Jakub, Manivannan, Charu, Bhatt, Mehul, \& Schultz, Carl. 2021. Embodied 3D isovists: A method to model the visual perception of space. Environment and Planning B: Urban Analytics and City Science, 48(8), 2307-2325.

Kuliga, Saskia F., Nelligan, Benjamin, Dalton, Ruth C., Marchette, Steven, Shelton, Amy L., Carlson, Laura, \& Hölscher, Christoph. 2019. Exploring Individual Differences and Building Complexity in Wayfinding: The Case of the Seattle Central Library. Environment and Behavior, 51(5), 622 -665.

Kurose, Shigeyuki, Borgers, Aloys W J, \& Timmermans, Harry J P. 2001. Classifying pedestrian shopping behaviour according to implied heuristic choice rules. Environment and Planning B: Planning and Design, 28(3), 405-418.

Lau, Stephen S.Y., \& Zhang, Qianning. 2015. Genesis of a Vertical City in Hong Kong. International Journal of High-Rise Buildings, 4(2), 117-125.

Li, Hengshan, Thrash, Tyler, Hölscher, Christoph, \& Schinazi, Victor R. 2019. The effect of crowdedness on human wayfinding and locomotion in a multi-level virtual shopping mall. Journal of Environmental Psychology, 65(July).

Lu, Yi, \& Ye, Yu. 2019. Can people memorize multilevel building as volumetric map? A study of multilevel atrium building. Environment and Planning B: Urban Analytics and City Science, 46(2), 225-242.

Macgregor, J. N., \& Ormerod, T. 1996. Human performance on the traveling salesman problem. Perception and Psychophysics, 58(4), 527-539.

MacGregor, James N. 2015. Effects of cluster location and cluster distribution on performance on the traveling salesman problem. Attention, Perception, and Psychophysics, 77(7), 2491-2501.

MacGregor, James N. 2017. Heuristics for comparing the lengths of completed E-TSP tours: Crossings and areas. Journal of Problem Solving, 10(1), 16-23.

Macgregor, James N, \& Chu, Yun. 2011. Human performance on the traveling salesman problem and Related Problems: A Review. The Journal of Problem Solving, 3(2).

Makowski, Dominique, Ben-Shachar, Mattan S, \& Lüdecke, Daniel. 2019a. bayestestR: Describing Effects and their Uncertainty, Existence and Significance within the Bayesian Framework. Journal of Open Source Software, 4(40), 1541.

Makowski, Dominique, Ben-Shachar, Mattan S., Chen, S. H.Annabel, \& Lüdecke, Daniel. 2019b. Indices of Effect Existence and Significance in the Bayesian Framework. Frontiers in Psychology, 10(December), $1-14$.

Manley, E J, Addison, J D, \& Cheng, T. 2015. Shortest path or anchor-based route choice: A large-scale empirical analysis of minicab routing in London. Journal of Transport Geography, 43, 123-139.

McFadden, Daniel. 1978. Modeling the choice of residential location. Transportation Research Record.

Meilinger, Tobias, Frankenstein, Julia, \& Bülthoff, Heinrich H. 2013. Learning to navigate: Experience versus maps. Cognition, 129(1), 24-30.

Menzel, E. 1973. Chimpanzee Spatial Memory Organization. Science, 182(4115), 943-945. 
Montello, Daniel R. 1993. Scale and multiple psychologies of space. Spatial information theory a theoretical basis for GIS, 311-321.

Montello, Daniel R., \& Pick, Herbert L. Jr. 1993. Integrating Knowledge of vertically aligned large-scale spaces. Environment \& Behavior, 25(4), 457-484.

Morey, Richard D, \& Rouder, Jeffrey N. 2018. BayesFactor: Computation of Bayes Factors for Common Designs.

Nardi, Daniele, Durgin, Frank H, Jeffery, Kate J, \& Weisberg, Steven M. 2012. How vertical spaces are perceived and represented. Cognition, 37, 354-367.

Passini, Romedi. 1984. Wayfinding in Architecture. New York: Van Nostrand Reinhold Company.

Phillips, F., \& Layton, O. 2010. The traveling salesman problem in the natural environment. Journal of Vision, 9(8), 1145-1145.

R Core Team. 2021. R: A Language and Environment for Statistical Computing.

Reynolds, Andrew M., Lihoreau, Mathieu, \& Chittka, Lars. 2013. A Simple Iterative Model Accurately Captures Complex Trapline Formation by Bumblebees Across Spatial Scales and Flower Arrangements. PLoS Computational Biology, 9(3), 1-10.

Saisa, Jouko, \& Garling, Tommy. 1987. Sequential Spatial Choices in the Large-scale Environment. Environment and Behavior, 19(5), 614-635.

Salazar Miranda, Arianna, Fan, Zhuangyuan, Duarte, Fabio, \& Ratti, Carlo. 2021. Desirable streets: Using deviations in pedestrian trajectories to measure the value of the built environment. Computers, Environment and Urban Systems, 86(March), 101563.

Seneviratne, P. N., \& Morrall, J. F. 1985. Analysis of factors affecting the choice of route of pedestrians. Transportation Planning and Technology, 10(2), 147-159.

Shaffer, Christopher A. 2014. Spatial foraging in free ranging bearded sakis: Traveling salesmen or Lévy walkers? American Journal of Primatology, 76(5), 472-484.

Starr, Ariel, \& Srinivasan, Mahesh. 2021. The future is in front, to the right, or below: Development of spatial representations of time in three dimensions. Cognition, 210(January), 104603.

Thibault, Guillaume, Pasqualotto, Achille, Vidal, Manuel, Droulez, Jacques, \& Berthoz, Alain. 2013. How does horizontal and vertical navigation influence spatial memory of multifloored environments? Attention, Perception \& Psychophysics, 75, 10-15.

van der Loo, M P J. 2014. The stringdist package for approximate string matching. The $\{R\}\left\{\int\right\}$ ournal, 6(1), 111-122.

Weisberg, Steven M, \& Newcombe, Nora S. 2015. How Do ( Some) People Make a Cognitive Map ? Routes , Places, and Working Memory. Journal of Experimental Psychology Learning Memory and Cognition.

Widdowson, Christopher, \& Wang, Ranxiao Frances. 2022. Human navigation in curved spaces. Cognition, 218(May 2021), 104923.

Wiener, J M, \& Tenbrink, T. 2008. Traveling Salesman Problem: The Human Case. KI: Themenheft KI und Kognition, 1(08), 18-22.

Wiener, J. M., Ehbauer, N. N., \& Mallot, H. A. 2009. Planning paths to multiple targets: Memory involvement and planning heuristics in spatial problem solving. Psychological Research, 73(5), 644-658.

Wiener, Jan M., \& Mallot, HA. 2003a. 'Fine-to-Coarse'Route Planning and Navigation in Regionalized Environments. Spatial cognition and computation, 3(4), 331-358.

Wiener, Jan M., Schnee, Alexander, \& Mallot, Hanspeter A. 2004. Use and interaction of navigation strategies in regionalized environments. Journal of Environmental Psychology, 24(4), 475-493.

Wiener, J.M., \& Mallot, Hanspeter A. 2003b. 'Fine-to-Coarse' Route Planning and Navigation in Regionalized Environments. Spatial Cognition and Computation, 3(4), 331-358.

Wilson, Paul N, Foreman, Nigel, Stanton, Danaë, \& Duffy, Hester. 2004. Memory for targets in a multilevel simulated environment: evidence for vertical asymmetry in spatial memory. Memory \& Cognition, 32(2), 283-297.

Zhang, Lingzhu, \& Chiaradia, Alain J F. 2020. Urban volumetrics: spatial complexity and wayfinding, extending space syntax to three dimensional space. 
Zwergal, Andreas, Schöberl, Florian, Xiong, Guoming, Pradhan, Cauchy, Covic, Aleksandar, Werner, Philipp, Trapp, Christoph, Bartenstein, Peter, La Fougère, Christian, Jahn, Klaus, Dieterich, Marianne, \& Brandt, Thomas. 2016. Anisotropy of Human Horizontal and Vertical Navigation in Real Space: Behavioral and PET Correlates. Cerebral Cortex, 26(11), 4392-4404. 